\title{
Interior Maximum Norm Estimates for Finite Element Methods
}

\author{
By A. H. Schatz and L. B. Wahlbin*
}

\begin{abstract}
Interior a priori error estimates in the maximum norm are derived from interior Ritz-Galerkin equations which are common to a class of methods used in approximating solutions of second order elliptic boundary value problems. The estimates are valid for a large class of piecewise polynomial subspaces used in practice, which are defined on quasi-uniform meshes.

It is shown that the error in an interior domain $\Omega_{1}$ can be estimated with the best order of accuracy that is possible locally for the subspaces used plus the error in a weaker norm over a slightly larger domain which measures the effects from outside of the domain $\Omega_{1}$.
\end{abstract}

0. Introduction. Let $D$ be a bounded domain in $R^{N}, N \geqslant 2$, with boundary $\partial D$. In order to illustrate the type of results we are seeking, consider a second order elliptic boundary value problem

$$
L u \equiv-\sum_{i, j=1}^{N} \frac{\partial}{\partial x_{j}}\left(a_{i j}(x) \frac{\partial u}{\partial x_{i}}\right)+\sum_{i=1}^{N} b_{i}(x) \frac{\partial u}{\partial x_{i}}+d(x) u=f \text { in } D,
$$

Some boundary conditions on $\partial D$.

Assume that this problem has a unique solution $u$. For $0<h<1$, let $S^{h}(D)$ be a one parameter family of finite element spaces. Many methods have been proposed in the literature for finding an approximation $u_{n}$ in $S^{h}(D)$ to the solution of special cases of the problem (0.1), (0.2), and we refer the reader to Bramble [2] for a survey of some of these procedures. Several of these methods differ only in the way they treat the boundary condition (0.2), but have the same interior equations. By this we mean that if we let $\Omega \subset \subset D$, and let $\xi^{n}(\Omega)$ denote the functions in $S^{h}(D)$ with compact supports in $\Omega$, then

$$
A\left(u-u_{h}, \chi\right)=0 \text { for all } \chi \in \stackrel{\circ}{S}^{h}(\Omega),
$$

where

$$
A(v, w)=\int_{\Omega}\left(\sum_{i, j=1}^{N} a_{i j} \frac{\partial v}{\partial x_{i}} \frac{\partial w}{\partial x_{j}}+\sum_{i=1}^{N} b_{i} \frac{\partial v}{\partial x_{i}} w+d v w\right) d x .
$$

In this paper we shall be concerned with deriving maximum norm estimates for $u-u_{n}$ on $\Omega_{1} \subset \subset \Omega$, where $u$ and $u_{n}$ are only required to satisfy the interior equation (0.3). This equation disregards what happens outside of $\Omega$. For example,

Received November 3, 1975; revised February 5, 1976 and March 8, 1976.

AMS (MOS) subject classifications (1970). Primary 65N30, 65N15.

* This work was supported by the National Science Foundation. 
it disregards the behavior of $u$ outside of $\Omega$, and the particular way a method handles the boundary conditions, in fact, what these boundary conditions are.

We shall now briefly describe some recent work on error estimates for Galerkin methods and then state and discuss our main result. Typically, let $S^{h}(\Omega) \subseteq W_{\infty}^{1}(\Omega)$ consist of the restrictions to $\Omega$ of a given class of piecewise polynomials defined on a quasi-uniform partition which covers $\Omega$. Let $r \geqslant 2$ denote the optimal order of $h$ (the "size" of the partition) to which functions in $S^{h}(\Omega)$ can approximate functions locally in $L_{q}$ norms. It was shown in Nitsche and Schatz [16] that if $(0.3)$ holds, then for $1 \leqslant q \leqslant \infty$ and $p \geqslant 0$,

$(0.5)\left\|u-u_{h}\right\|_{L_{2}\left(\Omega_{1}\right)} \leqslant C\left(h\left\|_{u}-\chi\right\|_{H^{1}(\Omega)}+\left\|u-u_{h}\right\|_{w_{q}^{-p}(\Omega)}\right)$ for any $\chi \in S^{h}(\Omega)$.

Here $\|\cdot\|_{W_{q}^{-p}(\Omega)}$ is the dual norm to that of $\stackrel{\circ}{W}_{q}^{p}, 1 / q+1 / q^{\prime}=1$.

Interior maximum norm estimates, in cases where the spaces $S^{h}$ are defined on uniform ("regular", "translation invariant") meshes, were given in Bramble, Nitsche and Schatz [3], Bramble and Schatz [5], Bramble and Thomée [6], and Strang and Fix [20]. Global maximum norm estimates for Dirichlet's problem were discussed in Ciarlet and Raviart [9]. Recently, quasi-optimal global estimates for general quasiuniform meshes have been obtained. The one-dimensional case of two point boundary value problems was given in Wheeler [21] in the context of continuous, but not continuously differentiable, piecewise polynomial spaces (and then without the assumption of quasi-uniformity), and the general case occurs in Douglas, Dupont and Wahlbin [10]. In Scott [18], the Neumann problem was treated for the equation $-\Delta u+u=$ $f$ and $N=2$. The Dirichlet problem for $-\Delta u=f$ in the case of $N=2$ and $r=2$ was discussed in Natterer [14], and for $N$ arbitrary and $r \geqslant 3$ announced in Nitsche [15]. In [15] and [18] the authors, in their respective situations, obtained the estimate

$$
\left\|u-u_{h}\right\|_{L_{\infty}(D)} \leqslant C h\left(\ln \frac{1}{h}\right)^{\bar{r}}\|u-\chi\|_{w_{\infty}^{1}(D)} \quad \text { for any } \chi \in S^{h}(D),
$$

where

$$
\bar{r}= \begin{cases}1 & \text { for } r=2, \\ 0 & \text { for } r \geqslant 3 .\end{cases}
$$

Let us return for a moment to estimates in $L_{2}$. If $u \in L_{2}(\Omega)$ and $S^{h}(\Omega) \subseteq$ $H^{2}(\Omega)$, then one can show that the estimate $(0.5)$ may be replaced by

(0.7) $\left\|u-u_{h}\right\|_{L_{2}\left(\Omega_{1}\right)} \leqslant C\left(\|u-\chi\|_{L_{2}(\Omega)}+\left\|u-u_{h}\right\|_{w_{q}^{-p}(\Omega)}\right)$ for any $\chi \in S^{h}(\Omega)$.

A proof of this will be presented in Appendix 2 for coercive forms $A$. However, the estimate (0.7) does not hold true if $S^{h}(\Omega) \nsubseteq H^{2}(\Omega)$. For example, if the $S^{h}(\Omega)$ are piecewise linear functions on triangles in the plane, then the equation $(0.3)$ does not in general make sense for $u \in L_{2}(\Omega)$.

In the present paper we shall show, for $S^{h}$ defined on quasi-uniform partitions, that (see Theorem 5.1 for the precise assumptions) 


$$
\left\|u-u_{h}\right\|_{L_{\infty}\left(\Omega_{1}\right)} \leqslant C\left(\left(\ln \frac{1}{h}\right)^{\bar{r}}\left\|_{u}-\chi\right\|_{L_{\infty}(\Omega)}+\left\|u-u_{h}\right\|_{w_{\bar{q}} p(\Omega)}\right)
$$

$$
\text { for any } \chi \in S^{h}(\Omega) \text {. }
$$

Note that by partial integration over each element, the equation (0.3) makes sense for $S^{h}(\Omega)$ as above and $u$ continuous on $\Omega$. The estimate $(0.8)$ has been previously proved in Bramble and Schatz [5] in the case that the spaces $S^{h}$ are smooth splines on a uniform mesh and $r \geqslant 3$. Their proof relies on Fourier methods, which are not available in the general quasi-uniform case.

Comparing the estimate $(0.8)$ to $(0.6)$, we have reduced the local smoothness requirements on the solution $u$, and we have also given estimates when $r=2$ and $N$ $\geqslant 3$.

The estimate $(0.8)$ essentially says that, except for the factor $\ln (1 / h)$ when $r=$ 2 , the Galerkin method is "locally bounded" in $L_{\infty}$. This is important since the estimate is then applicable to a large class of problems for which the solutions are not smooth in the interior. For example, if $u \in W_{\infty}^{s}(\Omega)$ for $0<s \leqslant r$, then the first term on the right of $(0.8)$ can be replaced by $C h^{s}(\ln (1 / h))^{\bar{r}}$; and the influences of the smoothness of $u$ outside of $\Omega$, and of the treatment of the boundary conditions in the approximate procedure, are contained in the second term. It is often the case that for a particular boundary value problem and approximation method an estimate of the form

$$
\left\|u-u_{n}\right\|_{w_{2}^{2-r}(D)} \leqslant C h^{s}\|u\|_{w_{2}^{\max }(1,2-r+s)}(D)
$$

holds. (For a survey of some methods satisfying this inequality, see Bramble and Osborn [4]. In more special cases, cf. (0.7), the norm on the right-hand side can be replaced by the norm in $W_{2}^{\max (0,2-r+s)}(D)$.) In this case we have that if $u \in W_{\infty}^{s}(\Omega)$ $\cap W_{2}^{\max (1,2-r+s)}(D)\left(\right.$ or $\left.u \in W_{\infty}^{s}(\Omega) \cap W_{2}^{\max (0,2-r+s)}(D)\right)$, then

$$
\left\|u-u_{h}\right\|_{L_{\infty}\left(\Omega_{1}\right)} \leqslant C h^{s}\left(\ln \frac{1}{h}\right)^{\bar{r}} \text { for } 0<s \leqslant r .
$$

In Section 6 (Theorem 6.1) we consider a model problem, namely the Neumann problem on a smooth domain, and derive pointwise estimates for the Green's function (cf. Bramble and Schatz [5] for a special case). Briefly, if $G^{(y)}(x)$ denotes the Green's function with singularity at $y, G_{h}^{(y)}(x)$ the usual Galerkin approximation, and if $x \in \Omega_{2}, y \in \Omega_{1}$ with $\Omega_{1} \subset \subset \Omega_{2}$, then

$$
\left|G^{(y)}(x)-G_{h}^{(y)}(x)\right| \leqslant C \frac{h^{r}(\ln (|x-y| / h))^{\bar{r}}}{|x-y|^{N-2+r}}
$$

provided $|x-y| \geqslant C h$. Except for the logarithmic factor when $r=2$, these estimates are "locally optimal". In Theorem 6.1 we also derive an estimate for the error when $|x-y| \leqslant C h$. As is well known, cf. [5], estimates of the above type can be used to derive error estimates when the right-hand side $f$ in $(0.1)$ is nonsmooth.

We shall now give a rough outline of the main steps in our analysis leading to the result (0.8). Assume that the form $A$ is coercive, cf. (4.1). (The proof for non- 
coercive forms follows from this case via an argument involving a compact perturbation of the elliptic operator, see Appendix 1.) Let $x_{0} \in \bar{\Omega}_{1}$ be such that

$$
\sup _{x \in \Omega_{1}}\left|\left(u-u_{h}\right)(x)\right|=\left|\left(u-u_{h}\right)\left(x_{0}\right)\right| .
$$

Let $D \subset \subset \Omega$ be a sphere with center at $x_{0}$, and $\omega$ a smooth cut-off function which is 1 in a neighborhood of $x_{0}$ and has compact support in $D$. We set $\tilde{u}=\omega u$ and let $\widetilde{u}_{h} \in S^{h}(D)$ satisfy

$$
A\left(\tilde{u}-\tilde{u}_{h}, \chi\right)=0 \quad \text { for } \chi \in S^{h}(D) \text {. }
$$

Here $\tilde{u}_{h}$ can be thought of as the approximate solution of a Neumann problem with right-hand side equal to $L \tilde{u}$. Taking $w_{n}=\tilde{u}_{n}-u_{n}$, we have

$$
\left(u-u_{h}\right)\left(x_{0}\right)=\left(\tilde{u}-\tilde{u}_{h}\right)\left(x_{0}\right)+w_{h}\left(x_{0}\right),
$$

so that the error $u-u_{n}$ consists of two parts, namely:

(i) The error $\tilde{u}-\tilde{u}_{n}$ in a projection for a "localized" $u$. Lemma 5.1 will in particular imply that

$$
\left|\left(\tilde{u}-\tilde{u}_{h}\right)\left(x_{0}\right)\right| \leqslant C(\ln 1 / h)^{\bar{r}}\|\tilde{u}-\chi\|_{L_{\infty}(D)} \text { for } \chi \in S^{h}(D) .
$$

(ii) The function $w_{h} \in S^{h}(D)$ which satisfies $A\left(w_{h}, \chi\right)=0$ for $\chi \in S^{h}(D)$ having support in a small neighborhood of $x_{0}$. In Lemma 5.2 we shall show that

$$
\left|w_{h}\left(x_{0}\right)\right| \leqslant C\left\|w_{h}\right\|_{w_{q}^{-p}(D)} .
$$

The desired result now follows from $(0.9),(0.10)$ and $(0.11)$ since

$$
\left\|w_{h}\right\|_{w_{q}^{-p}(D)} \leqslant\left\|\tilde{u}-\tilde{u}_{h}\right\|_{L_{\infty}(D)}+\left\|u-u_{h}\right\|_{w_{q}^{-p}(D)} .
$$

Here the first term on the right is again estimated via Lemma 5.1 .

The proofs of (0.10) and (0.11) rely on the fundamental Lemma 5.3. Briefly, Lemma 5.3 is concerned with error estimates in $L_{1}$-based norms for the Galerkin approximation $v_{h}$ of functions $v$ which satisfy an elliptic differential equation with righthand side $\varphi \in \mathcal{C}^{\infty}$ whose support is contained in a sphere $D_{h}$ of radius $C h$. For our purposes, any such $v$ may be thought of, after proper normalization, as a smoothedout Green's function. In Scott [18] maximum norm estimates were obtained by estimating the Green's function in $W_{1}^{1}$; that work motivated some of our considerations. In Lemma 5.3 we shall show that

$$
\left\|v-v_{h}\right\|_{w_{1}^{1}(D)} \leqslant C h^{N / 2+1}(\ln 1 / h)^{\bar{r}} \|_{\varphi \|_{L_{2}\left(D_{h}\right)}}
$$

and for $D_{I} \subset \subset D$,

$$
\left\|v-v_{h}\right\|_{w_{1}^{2, h}\left(D_{I}\right)} \leqslant C h^{N / 2}(\ln 1 / h)^{\bar{r}}\left\|_{\varphi}\right\|_{L_{2}\left(D_{h}\right)},
$$

where $W_{1}^{2, n}\left(D_{I}\right)$ denotes the piecewise norm relative to the element partition.

We shall now indicate how (0.10) follows from the estimates $(0.12)$ and $(0.13)$. 
It suffices to prove it for $\chi=0$. Instead of representing the error directly in terms of the Green's functions, as was done in Scott [18], we proceed as follows. Taking $x_{0}$ to be the center of $D_{h}$ we have, using inverse properties, that

$$
\left|\left(\tilde{u}-\tilde{u}_{h}\right)\left(x_{0}\right)\right| \leqslant C\left\{\|\tilde{u}\|_{L_{\infty}\left(D_{h}\right)}+h^{-N / 2}\left\|\tilde{u}-\tilde{u}_{h}\right\|_{L_{2}\left(D_{h}\right)}\right\} .
$$

We then employ a duality argument in order to estimate the second term on the right-hand side:

$$
\begin{aligned}
\left\|\tilde{u}-\tilde{u}_{h}\right\|_{L_{2}\left(D_{h}\right)} & =\sup _{\varphi \in \dot{C}^{\infty}\left(D_{h}\right)} \frac{\left(\tilde{u}-\tilde{u}_{h}, \varphi\right)}{\|\varphi\|_{L_{2}\left(D_{h}\right)}} \\
& =\sup _{\varphi \in \dot{C}^{\infty}\left(D_{h}\right)} \frac{A\left(\tilde{u}-\tilde{u}_{h}, v\right)}{\|\varphi\|_{L_{2}\left(D_{h}\right)}}=\sup _{\varphi \in \dot{C}^{\infty}\left(D_{h}\right)} \frac{A\left(\tilde{u}, v-v_{h}\right)}{\|\varphi\|_{L_{2}\left(D_{h}\right)}} .
\end{aligned}
$$

Integrating by parts over each element and taking care of the boundary terms via the trace inequality A.0 of Section 2, one deduces with $D_{I}$ approximately equal to supp $\omega$,

$\left\|\tilde{u}-\tilde{u}_{h}\right\|_{L_{2}\left(D_{h}\right)} \leqslant \sup _{\varphi \in \dot{C}^{\infty}\left(D_{h}\right)} C \frac{\|\tilde{u}\|_{L_{\infty}(D)}}{\|\varphi\|_{L_{2}\left(D_{h}\right)}}\left(h^{-1}\left\|_{v}-v_{h}\right\|_{w_{1}^{1}\left(D_{I}\right)}+\left\|v-v_{h}\right\|_{w_{1}^{2, h}\left(D_{I}\right)}\right)$.

The result (0.10) now follows using (0.12) and (0.13).

.The proof of $(0: 11)$ proceeds along somewhat similar lines.

To prove ( 0.12$)$ (from which (0.13) follows) we start by subdividing $D$, which for simplicity we assume has radius 1 and center at $x_{0}$, into annuli

$$
\Omega_{j}=\left\{x\left|2^{-(j-1)} \leqslant\right| x-x_{0} \mid \leqslant 2^{-j}\right\}, \quad j=0, \ldots, J,
$$

and a sphere $\Omega_{h}$ of radius $C_{*} h=2^{-J}$, centered at $x_{0}$, so that $D=\Omega_{h} \cup\left(\cup_{j=0}^{J} \Omega_{j}\right)$. Let $e=v-v_{h}$. We have

$$
\|e\|_{w_{1}^{1}(D)}=\sum_{j=0}^{J}\|e\|_{w_{1}^{1}\left(\Omega_{j}\right)}+\|e\|_{w_{1}^{1}\left(\Omega_{h}\right)} .
$$
We estimate each $\|e\|_{W_{1}^{1}\left(\Omega_{j}\right)}\left(\right.$ and $\left.\|e\|_{W_{1}^{1}\left(\Omega_{h}\right)}\right)$ separately. Using the Cauchy-Schwarz
inequality

$$
\|e\|_{w_{1}^{1}\left(\Omega_{j}\right)} \leqslant C d_{j}^{N / 2}\|e\|_{1, \Omega_{j}}
$$

where $d_{j}=2^{-j}$. We apply Lemma 3.2, or Lemma 4.4 in the case of $j=0$, both of which are generalizations of the interior $H^{1}$ estimates of Nitsche and Schatz [16], to obtain

$$
\|e\|_{w_{1}^{1}\left(\Omega_{j}\right)} \leqslant C\left(d_{j}^{N / 2} h^{r-1}\left\|_{u}\right\|_{w_{2}^{r}\left(\Omega_{j}^{1}\right)}+d_{j}^{-1}\|e\|_{L_{1}\left(\Omega_{j}^{1}\right)}\right),
$$

where $\Omega_{j}^{1}=\left(\Omega_{j-1} \cup \Omega_{j} \cup \Omega_{j+1}\right) \cap D$. We then sum and estimate the terms on the right-hand side, using in particular a "kickback" argument which involves choosing $C_{*}=2^{-J} / h$ sufficiently large. The details are quite technical. We wish to point out again, however, the use in the proof of $H^{1}$-estimates which depend on distances, roughly equal to $d_{j}$, between domains $\Omega_{j}$ and $\Omega_{j}^{1}$, where also $d_{j}$ is approximately the 
distance from $\partial \Omega_{j}$ to $x_{0}$. This idea is related to estimating norms on annuli which are weighted in terms of their distance to $x_{0}$. The papers Natterer [14] and Nitsche [15] use weighted norms to prove their results, and similarly Scott [18] employs weighted norms implicitly. The techniques applied in these papers have much in common with the ideas of Nitsche and Schatz [16], as do the techniques of the present paper.

In a forthcoming paper we shall adapt the local techniques developed here to analyze the error in approximating the solution of a Dirichlet problem on a plane polygonal domain. Some of these results were presented at the SYNSPADE III conference at the University of Maryland in May 1975.

An outline of this paper is as follows. In Section 1 we introduce some notation. Assumptions on the finite element spaces are listed in Section 2. In Section 3 we recall the interior $H^{1}$-estimates of [16], and extend them somewhat. Section 4 is concerned with some estimates for an auxiliary Neumann problem. Then, in Section 5 , we prove the estimate $(0.8)$ in the case of coercive forms $A$; the proof for noncoercive forms is given in Appendix 1. Lastly, in Section 6, we give the application to pointwise error estimates for the Green's function.

1. Notation. Throughout this paper, $c$ and $C$ will denote positive generic constants. Let $\Omega$ be a bounded domain in $R^{N}$. For $1 \leqslant q \leqslant \infty$ we denote by $L_{q}(\Omega)$ the usual Banach spaces with norm $\|\cdot\|_{L_{q}(\Omega)}$. For $m$ a nonnegative integer, $W_{q}^{m}(\Omega)$ will be the usual Sobolev spaces with norms

$$
\|v\|_{w_{q}^{m}(\Omega)}= \begin{cases}\left(\sum_{j=0}^{m}|v|_{w_{q}^{q}(\Omega)}\right)^{1 / q} & \text { for } 1 \leqslant q<\infty, \\ \max _{j=0, \ldots, m}|v|_{w_{\infty}^{j}(\Omega)} & \text { for } q=\infty .\end{cases}
$$

Here $|\cdot| w_{q}^{j}(\Omega)$ denotes the seminorms

$$
|v|_{w_{q}^{j}(\Omega)}= \begin{cases}\left(\sum_{|\alpha|=j}\left\|D^{\alpha} v\right\|_{L_{q}(\Omega)}^{q}\right)^{1 / q} & \text { for } 1 \leqslant q<\infty, \\ \max _{|\alpha|=j}\left\|D^{\alpha} v\right\|_{L_{\infty}(\Omega)} & \text { for } q=\infty\end{cases}
$$

We define the spaces $\stackrel{\circ}{W}_{q}^{m}(\Omega)$ as the completion of $\mathcal{C}^{\infty}(\Omega)$ in the norm of $W_{q}^{m}(\Omega)$. If $q=2$, we shall write $W_{2}^{m}(\Omega)=H^{m}(\Omega)$ and use the symbols $\|\cdot\|_{m, \Omega}$ and $|\cdot|_{j, \Omega}$ for the norm and seminorm, respectively.

For $m$ a negative integer we use the notation $\|\cdot\|_{w_{q}^{m}(\Omega)}$ for the norm dual to $\stackrel{\circ}{W}_{q^{\prime}}^{-m}(\Omega), 1 / q+1 / q^{\prime}=1$, i.e.,

$$
\|v\|_{w_{q}^{m}(\Omega)}=\sup _{\varphi \in \check{c}^{\infty}(\Omega)} \frac{(v, \varphi)}{\|\varphi\|_{w_{q^{\prime}}^{-m}(\Omega)}}
$$

where $(v, w)=\int_{\Omega} v w d x$. For $q=2$ we again write $\|\cdot\|_{m, \Omega}$ for $\|\cdot\|_{w_{2}^{m}(\Omega)}=$ 
$\|\cdot\|_{H^{m}(\Omega)}$. We note that if $\Omega_{1} \subseteq \Omega_{2}$, then

$$
\|v\|_{w_{q}^{m}\left(\Omega_{1}\right)} \leqslant\|v\|_{w_{q}^{m}\left(\Omega_{2}\right)} \text {. }
$$

In the proof of Lemma 3.2 we shall also need the following result.

LemmA 1.1. Let $1 \leqslant q \leqslant 2$ and $p \geqslant 0$. Furthermore, let $\Omega_{j}, j=1, \ldots, J$, be disjoint sets and $\Omega=\bigcup_{1}^{J} \Omega_{j}$. Then

$$
\sum_{j=1}^{J}\|v\|_{w_{q}^{2}\left(\Omega_{j}\right)} \leqslant\|v\|_{w_{q}^{2}(\Omega)}^{-p} .
$$
$\begin{aligned} & \text { Proof. } \text { Let } \varphi_{j} \in \mathcal{C}_{0}^{\infty}\left(\Omega_{j}\right), j=1, \ldots, J \text {, be such that }\left\|\varphi_{j}\right\|_{W_{q}^{p}\left(\Omega_{j}\right)}=1 \text {, where } \\ & 1 / q+1 / q^{\prime}=1 \text {. Set }\end{aligned}$

$$
\theta_{j}=\|v\|_{w_{q}^{-p}\left(\Omega_{j}\right)} \text { and } \varphi=\sum_{j=1}^{J} \cdot \theta_{j}^{q-1} \varphi_{j}
$$

note that $\varphi \in C_{0}^{\infty}(\Omega)$. We have

$$
\|v\|_{W_{q}^{q}(\Omega)}^{q} \geqslant\left(\frac{(v, \varphi)}{\|\varphi\|_{w_{q}^{p},(\Omega)}}\right)^{q}=\frac{\left(\sum_{j=1}^{J} \theta_{j}^{q-1}\left(v, \varphi_{j}\right)\right)^{q}}{\left(\sum_{j=1}^{J} \theta_{j}^{q}\right)^{q-1}} .
$$

Taking the supremum of the right-hand side over $\varphi_{j}$, we deduce that

$$
\|v\|_{w_{q}^{q}(\Omega)} \geqslant \sum_{j=1}^{J} \theta_{j}^{q} .
$$

The lemma now follows since the norm in the sequence space $l_{q}$ majorizes the norm in $l_{2}$ for $1 \leqslant q \leqslant 2$.

We shall also make use of spaces defined relative to partitions of $\Omega$. Let $0<h$ $<1$ be a parameter, and for each $h$ let $\tau_{i}^{n}, 0 \leqslant i \leqslant I(h)$ be a finite number of disjoint open sets such that $\Omega \subseteq \bigcup_{i=0}^{I(n)} \bar{\tau}_{i}^{n}$. The sets $\tau_{i}^{n} \cap \Omega$ induce a partition of $\Omega$, and relative to each such partition, we define $W_{q}^{m, n}(\Omega)\left(C^{m, n}(\Omega)\right)$ as the space consisting of those functions which belong to $W_{q}^{m}\left(\tau_{i}^{h} \cap \Omega\right)\left(C^{m}\left(\tau_{i}^{h} \cap \Omega\right)\right), 0 \leqslant i \leqslant I(h)$. We introduce the seminorms

$$
|v|_{w_{q}^{m, n}(\Omega)}= \begin{cases}\left(\sum_{i=0}^{I(n)}|v|_{w_{q}^{m}\left(\tau_{i}^{h} \cap \Omega\right)}^{q}\right)^{1 / q} & \text { for } 1 \leqslant q<\infty, \\ \max _{i=0, \ldots, I(h)}|v|_{w_{\infty}^{m}\left(\tau_{i}^{h} \cap \Omega\right)} & \text { for } q=\infty,\end{cases}
$$

and the corresponding norms $\|\cdot\|_{w_{q}^{m, n}(\Omega)}$. Note that if $v \in W_{q}^{m}(\Omega)$, then $\|v\|_{w_{q}^{m, h}(\Omega)}$ $=\|v\|_{w_{q}^{m}(\Omega)}$.

2. The Finite Element Spaces. Let $\Omega \subset \subset R^{N}$ be fixed throughout this section. We shall describe a class of families of finite-dimensional spaces which have properties that are shared by many quasi-uniform finite element spaces used in practice to approximate solutions of partial differential equations. 
For each $0<h<1, S^{h}(\Omega)$ will denote a finite-dimensional subspace of $W_{\infty}^{1}(\Omega)$ $\cap C^{2, h}(\Omega)$. Our first assumption relates to the geometry of the partitioning sets $\tau_{i}^{n}$. We shall assume that a certain trace inequality holds on each of the $\tau_{i}^{h}$.

A.0. There exists a constant $C$ such that for $0<h<1$, and any $f \in W_{1}^{2}\left(\tau_{i}^{h}\right)$, $i=0, \ldots, I(h)$,

$$
\int_{\partial \tau_{i}^{n}}|\nabla f| d \sigma \leqslant C\left\{\frac{1}{h}|f|_{w_{1}^{1}\left(\tau_{i}^{n}\right)}+|f|_{w_{1}^{2}\left(\tau_{i}^{n}\right)}\right\} .
$$

We remark that the assumption A.0 is satisfied for a large class of partitions of $\Omega$. For example, it holds if the $\tau_{i}^{h}$ are taken to be $N$-simplices or $N$-dimensional parallelepipeds of diameter $c_{i} h, c_{i} \leqslant C$, provided the ratio of the diameter and the radius of the largest inscribed sphere is uniformly bounded. Briefly, to verify A.0 in these cases one maps each of the $\tau_{i}^{h}$ onto a standard domain. The inequality can then be proven, with $h=1$, using integration by parts. The desired inequality is then obtained by mapping back to $\tau_{i}^{h}$.

For $D \subseteq \Omega, S^{h}(D)$ is defined as the restriction of $S^{h}(\Omega)$ to $D$, and

$$
\stackrel{\circ}{S}^{h}(D)=\left\{\chi \mid \chi \in S^{h}(D) \text {, supp } \chi \subset \subset D\right\} .
$$

Let $r \geqslant 2$ be a given integer. We shall assume that there exist positive constants $C_{1}$, $C_{2}, C_{3}, C_{4}, k_{0}, \gamma$, and $0<h_{0}<1$ such that the spaces $S^{h}(\Omega)$ satisfy the following conditions A.1-A.4 for $0<h \leqslant h_{0}$.

A.1. Let $s=0,1$, or 2 and let $D_{1} \subset \subset D$ with $\operatorname{dist}\left(D_{1}, \partial D\right) \geqslant k_{0} h$ and $\operatorname{dist}(D, \partial \Omega) \geqslant k_{0} h$. Then for each $v$ there exists a $\chi \in S^{h}(D)$ such that

$$
\|v-\chi\|_{w_{q}^{t, h}\left(D_{1}\right)} \leqslant C_{1} h^{l-t}|v|_{w_{q}^{l}(D)} \text { for } 0 \leqslant t \leqslant s \leqslant l \leqslant r, 1 \leqslant q \leqslant \infty
$$

Furthermore, if $D_{2} \subset \subset D_{1}$ with $\operatorname{dist}\left(D_{2}, \partial D_{1}\right) \geqslant k_{0} h$ and supp $v \subseteq D_{2}$, then $\chi \in$ $\dot{S}^{n}\left(D_{1}\right)$.

A.2. Inverse Properties. Let $p \geqslant-1$ be an integer and $D_{1} \subset \subset D$ with $\operatorname{dist}\left(D_{1}, \partial D\right) \geqslant k_{0} h$. Then for $\chi \in S^{h}(D)$,

$$
\|\chi\|_{1, D_{1}} \leqslant C_{2} h^{-(p+1)}\left\|_{\chi}\right\|_{-p, D}
$$

$$
\|\chi\|_{w_{q}^{s, h}\left(D_{1}\right)} \leqslant C_{2} h^{t-s-N\left(1 / q_{1}-1 / q\right)}\left\|_{\chi}\right\|_{w_{q_{1}}^{t, h}(D)} \text { for } 0 \leqslant t \leqslant s \leqslant 2,1 \leqslant q_{1} \leqslant q \leqslant \infty
$$

A.3. Let $D_{1} \subset \subset D$ with $\operatorname{dist}\left(D_{1}, \partial D\right) \geqslant k_{0} h$, and let $\omega \in \mathcal{C}^{\infty}\left(D_{1}\right)$. Then for each $\chi \in S^{h}(D)$ there exists an $\eta \in S^{n}(D)$ satisfying

$$
\|\omega \chi-\eta\|_{1, D} \leqslant C_{3} h\|\omega\|_{w_{\infty}^{\gamma}\left(D_{1}\right)}\|\chi\|_{1, D_{1}} .
$$

Furthermore, let $D_{4} \subset \subset D_{3} \subset \subset D_{2} \subset \subset D_{1}$ with $\operatorname{dist}\left(D_{4}, \partial D_{3}\right) \geqslant k_{0} h$ and $\operatorname{dist}\left(D_{3}, \partial D_{2}\right) \geqslant k_{0} h$. Then if $\omega \equiv 1$ on $D_{2}$ we have $\eta \equiv \chi$ on $D_{3}$ and

$$
\|\omega \chi-\eta\|_{1, D} \leqslant C_{3} h\|\omega\|_{W_{\infty}^{\prime}\left(D_{1}\right)}\|\chi\|_{1, D_{1} \backslash D_{4}} .
$$


We shall finally make the assumption that if a sphere or radius $d$ in $\Omega$ is transformed by similarity to a sphere of unit size, then the transformed finite element space satisfies A.1, A.2, and A.3 with $h$ replaced by $h / d$ and with the constants occurring the same as before.

A.4. Let $D_{d} \subset \subset \Omega$ be a sphere of radius $d \geqslant C_{4} h$ with center at $x_{0}$. The linear transformation $y=\left(x-x_{0}\right) / d$ takes $D_{d}$ into a sphere $\widetilde{D}$ and $S^{h}\left(D_{d}\right)$ into a new function space $S(\widetilde{D})$. Then $S(\widetilde{D})$ satisfies A.1, A.2, and A.3 with $h$ replaced by $h / d$. Furthermore, the constants occurring in A.1, A.2, and A.3 remain unchanged, in particular, independent of $d$.

Examples of finite element spaces for which the above assumptions obtain are:

(i) The Lagrange and Hermite elements, cf. [8], which include, for example, the restriction to $\Omega$ of piecewise linear elements, defined on a sequence of quasi-uniform simplicial partitions.

(ii) The plane triangular elements of Bramble and Zlámal [7].

(iii) The restriction to $\Omega$ of tensor products of one-dimensional piecewise polynomials, cf. [3].

In these examples the properties A.1-A.3 are well known, cf. [11] and [19] in the case of A.1, and [3] and [16] for A.3. That A.4 holds with respect to A.1 and A.2 follows via a scaling argument. For A.3, on the transformed spaces $S(\widetilde{D})$, one has to consider the original proofs. In the above examples, these proofs give (2.3) and (2.4) with $h$ replaced by $h / d$.

We shall now state two easy consequences of our assumptions.

Proposition 2.1. Assume A.1, and let $R_{0}>0$ be fixed. There exists a constant $C$ such that the following holds:

Let $D \subset \subset \Omega$ be a sphere of radius $R \geqslant R_{0}$ with $\operatorname{dist}(D, \partial \Omega) \geqslant 2 k_{0} h$. Let $s=$ 0,1 or 2 . Then for each $v$ there exists $a \chi \in S^{h}(D)$ such that

$$
\|v-\chi\|_{w_{q}^{t, h}(D)} \leqslant C h^{l-t}\left\|_{v}\right\|_{w_{q}^{l}(D)} \text { for } 0 \leqslant t \leqslant s \leqslant l \leqslant r, 1 \leqslant q \leqslant \infty .
$$

Proof. The function $v$ may be extended coniinuously in all $W_{q}^{l}$ norms to a sphere $D_{1}$ of radius $2 R$ such that

$$
\|v\|_{w_{q}^{l}\left(D_{1}\right)} \leqslant C\left(R_{0}\right)\|v\|_{w_{q}^{l}(D)} .
$$

The result now follows from A.1.

We shall need a way of cutting down functions in the finite element space to have compact support.

Proposition 2.2. Let A.3 hold and let $D_{3} \subset \subset D_{2} \subset \subset D_{1} \subset \subset D \subset \subset \Omega$. There exists a constant $C$ such that if $h$ is sufficiently small the following holds:

For each $\chi \in S^{h}(D)$ there exists an $\eta \in \xi^{h}\left(D_{1}\right)$ with $\eta \equiv \chi$ on $D_{2}$ and

$$
\|\chi-\eta\|_{1, D} \leqslant C\left\|_{\chi}\right\|_{1, D \backslash D_{3}} .
$$

Proof. Let $\omega \in \mathcal{C}^{\infty}\left(D_{4}\right)$ and $\omega \equiv 1$ on $D_{5}$, where $D_{2} \subset \subset D_{5} \subset \subset D_{4} \subset \subset D_{1}$. By A.3 we can find $\eta \in \xi^{n}\left(D_{1}\right)$ with $\eta \equiv \chi$ on $D_{2}$ such that 


$$
\|\omega \chi-\eta\|_{1, D} \leqslant C h\left\|_{\chi}\right\|_{1, D \backslash D_{3}} .
$$

Since $\|(1-\omega) \chi\|_{1, D} \leqslant C\left\|_{\chi}\right\|_{1, D \backslash D_{3}}$, we obtain the desired result by the triangle inequality.

In what follows we shall set $k=2 k_{0}$.

3. Interior Estimates in $H^{1}$. Consider the bilinear form $A$ of $(0.4)$ where, for simplicity, $a_{i j}, b_{i}$ and $d$ belong to $C^{\infty}(\bar{\Omega})$ and $a_{i j}=a_{i i}$. Assume that $A$ is uniformly elliptic on $\bar{\Omega}$, i.e., there exists a positive constant $C_{\mathrm{ell}}$ such that for all real vectors $\xi=\left(\xi_{1}, \ldots \xi_{N}\right)$ and $x \in \bar{\Omega}$,

$$
\sum_{i, j=1}^{N} a_{i j}(x) \xi_{i} \xi_{j} \geqslant C_{\mathrm{ell}} \sum_{i=1}^{N} \xi_{i}^{2}
$$

Let $u_{n} \in S^{h}(\Omega)$ be such that

$$
A\left(u-u_{h}, \chi\right)=0 \text { for all } \chi \in S^{n}(\Omega) .
$$

Interior estimates for $u-u_{n}$ in $H^{s}$ norms were given in [16]. Our investigations will rely on the following special case of a result from that paper.

Lemma 3.1. Assume that (3.1), A.1 (for $q=2$ ), (2.2a) of A.2 and (2.3) of A.3 hold. Let $p$ be a nonnegative integer and $D_{1} \subset \subset D \subset \subset \Omega$. There exist constants $h_{1}=h_{1}\left(p, \operatorname{dist}\left(D_{1}, \partial D\right)\right)$ and $C$ such that the following holds:

Let $u$ and $u_{h} \in S^{h}(\Omega)$ satisfy (3.2). Then for $0<h \leqslant h_{1}, 1 \leqslant q \leqslant \infty, s=0$ or 1 , and $1 \leqslant l \leqslant r$,

$$
\left\|u-u_{h}\right\|_{s, D_{1}} \leqslant C\left\{h^{l-s}|u|_{l, D}+\left\|u-u_{h}\right\|_{w_{q}^{-p}(D)}\right\} .
$$

For $D_{1}$ and $D$ concentric spheres of radii $R_{1}$ and $R$, the constant $C$ depends on $p$, and monotonically on $C_{\mathrm{ell}}^{-1},\left(R-R_{1}\right)^{-1},\left\|a_{i j}\right\|_{w_{\infty}^{\alpha}(\Omega)},\left\|b_{i}\right\|_{w_{\infty}^{\beta}(\Omega)}$ and $\|d\|_{w_{\infty}^{\gamma}(\Omega)}$ for some fixed positive integers $\alpha, \beta, \gamma$.

Lemma 3.1 was proved in [16] for $q=2$, but follows for all $q$ by use of Sobolev's lemma.

In particular, if $u_{n}$ satisfies $A\left(u_{n}, \chi\right)=0$ for all $\chi \in S^{n}(\Omega)$, then

$$
\left\|u_{h}\right\|_{s, D_{1}} \leqslant C\left\|u_{h}\right\|_{w_{q}^{-p}(D)} \text {. }
$$

In Lemma 3.1 the domains $D$ and $D_{1}$ were arbitrary but fixed. We shall need the precise behavior of the constants in (3.3) in the case when $D$ and $D_{1}$ may vary, and are possibly close, with $h$. We shall find this behavior by a scaling argument, using A.4, and applying Lemma 3.1 on domains of unit size.

Lemma 3.2. Let (3.1), A.1, A.2, A.3 and A.4 hold. Let $p$ be a nonnegative integer. There exist constants $C$ and $C_{5}$ such that the following holds:

Let $D_{1} \subset \subset D \subset \subset \Omega$ with $\operatorname{dist}(D, \partial \Omega) \geqslant k h$ and $\operatorname{dist}\left(D_{1}, \partial D\right)=d \geqslant C_{5} h$. If $u$ and $u_{h} \in S^{h}(\Omega)$ satisfy (3.2), then for $1 \leqslant q \leqslant 2$,

$$
\left\|u-u_{h}\right\|_{1, D_{1}} \leqslant C\left\{h^{r-1}|u|_{r, D}+d^{N(1 / 2-1 / q)-(p+1)}\left\|_{u}-u_{h}\right\|_{w_{q}^{-p}(D)}\right\} .
$$


Proof. We first note that if $d$ is of unit size, then (3.5) is a special case of (3.3). By (1.1), Lemma 1.1 and a covering argument it suffices to prove (3.5) with $D_{1}$ and $D$ taken as spheres of radii $d / 2$ and $d$, respectively, with centers at $x_{0}$. Assume $x_{0}=$ 0 and let $x$ denote the variable on $D$. Let $y=x / d$ be the new variable on the transformed regions $\widetilde{D}_{1}$ and $\widetilde{D}$; note that $\operatorname{dist}\left(\widetilde{D}_{1}, \partial \widetilde{D}\right)=1 / 2$. Set

$$
e(x)=u(x)-u_{n}(x)
$$

and

$$
E(y)=U(y)-U_{h}(y) \equiv u(y d)-u_{h}(y d)
$$

We have

$$
\|e\|_{1, D_{1}}^{2}=d^{N}\left(d^{-2}|E|_{1, \widetilde{D}_{1}}^{2}+|E|_{0, \widetilde{D}_{1}}^{2}\right)
$$

so that if $d \leqslant 1$, as may be assumed,

$$
\|e\|_{1, D_{1}} \leqslant d^{N / 2-1}\|E\|_{1, \widetilde{D}_{1}} .
$$

Next note that $E$ satisfies the relation

$$
A_{d}(E, \tilde{\chi})=0
$$

for $\tilde{\chi}$ in the transformed space, where with $\delta_{i}$ denoting differentiation with respect to $y_{i}$

$$
\begin{aligned}
A_{d}(v, w) \equiv \int_{\widetilde{D}}( & \sum_{i, j=1}^{N}\left(a_{i j}(y d) \delta_{i} v(y) \delta_{j} w(y)\right) \\
& \left.+\left(d \sum_{i=1}^{N}\left(b_{i}(y d) \delta_{i} v(y)\right)+d^{2} d(y d) v(y)\right) w(y)\right) d y
\end{aligned}
$$

This form is uniformly elliptic with the same modulus of ellipticity $C_{\text {ell }}$ as for the original form $A$, and the norms of derivatives of the coefficients are decreased compared to those of $A$. From (3.7), A.4 and Lemma 3.1 we deduce that for $h / d \leqslant h_{1}=$ $1 / C_{5}$,

$$
\|E\|_{1, \tilde{D}_{1}} \leqslant C\left(\left(\frac{h}{d}\right)^{r-1}|U|_{r, \widetilde{D}}+\|E\|_{W_{q}^{-p}(\tilde{D})}\right) .
$$

One easily finds that

$$
|U|_{j, \tilde{D}} \leqslant d^{-N / 2+j}|u|_{j, D}
$$

and using the definition of the $W_{q}^{-p}$ norm, we have for $d \leqslant 1$,

$$
\|E\|_{w_{q}^{-p}(\tilde{D})} \leqslant d^{-N / q-p}\left\|_{e}\right\|_{w_{q}^{-p}(D)}
$$

From (3.6), (3.8), (3.9), and (3.10) we obtain the inequality (3.5). This proves the lemma. 
4. An Auxiliary Neumann Problem. In order to prove our interior estimates we shall employ an auxiliary Neumann problem. In this section we state this problem and collect some facts relating to it.

Throughout this section we shall assume that if $D \subseteq \Omega$, then the form $A$ is coercive over $H^{1}(D)$, i.e., there exists a positive constant $c$ independent of $D$ such that

$$
c\|v\|_{1, D}^{2} \leqslant A(v, v) \text { for all } v \in H^{1}(D) .
$$

Here the region of integration for $A$ is $D$. In the sequel the appropriate region of integration will be clear from the context.

Let $D \subseteq \Omega$ be a sphere. Then for each $\varphi \in L_{2}(D)$ there exists a unique function $v$ in $H^{1}(D)$ such that

$$
A(v, w)=(\varphi, w) \text { for all } w \in H^{1}(D)
$$

This may be thought of as the variational formulation of a Neumann problem for a second order operator with the natural boundary conditions being the vanishing of the conormal derivative. The following a priori estimates are well known, cf. [17] .

Lemma 4.1. Assume that (3.1), (4.1), and (4.2) hold, let $1<q<\infty$, and let $m$ be a nonnegative integer. Then there exists a constant $C$ such that

$$
\|v\|_{w_{q}^{m+2}(D)} \leqslant C\left\|_{\varphi}\right\|_{w_{q}^{m}(D)} .
$$

We shall be particularly concerned with the problem (4.2) when $\varphi$ has small support. Let $D$ have radius 1 and center at the origin, and let

$$
D_{[a, b]}=\{x: a \leqslant|x| \leqslant b\} .
$$

Write $B_{\epsilon}$ for $D_{[0, \epsilon]}$. We have the following estimates.

LEMMA 4.2. There exists a constant $C$ such that if $v$ and $\varphi$ satisfy (4.2), then the following hold:

(i) If supp $\varphi \subseteq B_{\epsilon}$, then

$$
\begin{gathered}
\|v\|_{r, D[a, 2 a]} \leqslant C a^{-N / 2+2-r} \epsilon^{N / 2}\|\varphi\|_{0, B_{\epsilon}} \text { for } 1 / 4 \geqslant a \geqslant 2 \epsilon, r \geqslant 1, \\
\|v\|_{w_{1}^{r}\left(D \backslash B_{2 \epsilon}\right)} \leqslant C \epsilon^{2-r}(\ln 1 / \epsilon)^{\bar{r}}\left\|_{\varphi \|_{L_{1}\left(B_{\epsilon}\right)}, \quad r \geqslant 2,} \leqslant c\right\|_{w_{\infty}^{1}\left(D \backslash B_{2 \epsilon}\right)} \leqslant C \epsilon^{1-N}\left\|_{\varphi \|_{L_{1}\left(B_{\epsilon}\right)},} \leqslant C(l) \epsilon\right\|_{\varphi \|_{L_{1}\left(B_{\epsilon}\right)} .}
\end{gathered}
$$

(ii) If supp $\varphi \subseteq D_{[a, 2 a]}$, then

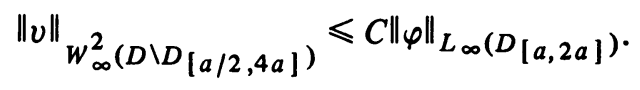

(iii) For $\varphi \in L_{1}(D)$,

$$
\|v\|_{W_{1}^{1}(D)} \leqslant C\|\varphi\|_{L_{1}(D)}
$$

Proof. We have 


$$
v(x)=\int_{D} G^{(x)}(y) \varphi(y) d y,
$$

where $G$ is the Green's function for the problem (4.2). By [12] and [13],

$$
\left|D_{x}^{\alpha} G^{(x)}(y)\right| \leqslant \begin{cases}C(1+|\ln | x-y \mid 1) & \text { for }|\alpha|=0, N=2, \\ C|x-y|^{2-N-|\alpha|} & \text { for } N \geqslant 3 \text { or } N=2,|\alpha|>0 .\end{cases}
$$

Let us show how this implies (4.3); the other estimates are proved in a similar fashion. For $x \in D_{[a, 2 a]}$ and $1 \leqslant|\alpha| \leqslant r$, we have

$$
\begin{aligned}
\left|D^{\alpha} v(x)\right| & \leqslant \int_{B_{\epsilon}}\left|D_{x}^{\alpha} G^{(x)}(y) \| \varphi(y)\right| d y \\
& \leqslant\left(\int_{B_{\epsilon}}|x-y|^{-2(N-2+|\alpha|)} d y\right)^{1 / 2}\|\varphi\|_{0, B_{\epsilon}} \leqslant C \epsilon^{N / 2} a^{-N+2-r}\|\varphi\|_{0, B_{\epsilon}} .
\end{aligned}
$$

The same estimate is obviously true for $|\alpha|=0$. Squaring and integrating with respect to $x$ we obtain the estimate (4.3).

The classical Ritz-Galerkin approximation $v_{h} \in S^{h}(D)$ to $v$ is defined by

$$
A\left(v-v_{h}, \chi\right)=0 \text { for all } \chi \in S^{h}(D) \text {. }
$$

We recall the following error estimates, cf. [1] and Proposition 2.1.

Lemma 4.3. Assume (3.1), (4.1) and A.1 (for $q=2)$. There exists a constant $C$ such that the following holds:

Let $v$ and $v_{h} \in S^{h}(D)$ satisfy (4.10). Then for $2-r \leqslant s \leqslant 1,1 \leqslant l \leqslant r$,

$$
\left\|_{v}-v_{h}\right\|_{s, D} \leqslant C h^{l-s}\left\|_{u}\right\|_{l, D} .
$$

We shall also need a local estimate near the boundary, corresponding to Lemma 3.1, for the projection with respect to the form $A$. The proof will follow along the lines of Lemma 5.1 in [16] with some simplifications due to the fact that $A$ is coercive. Let $D_{0} \subset \subset D, D_{0}^{\prime}=D W_{0}$, and define

$$
\stackrel{\complement}{S}^{h}\left(D_{0}^{\prime}\right)=\left\{\chi \in S^{h}(D), \chi \equiv 0 \text { in a neighborhood of } D_{0}\right\} .
$$

Lemma 4.4. Assume that (3.1), (4.1), A.1 (for $q=2$ ), (2.2.a) of A.2 and A.3 hold. Let $D_{0} \subset \subset D_{1} \subset \subset D \subset \subset \Omega$ be concentric spheres, and $D_{i}^{\prime}=D D_{i}, i=0,1$. There exists a constant $C$ such that for $h$ sufficiently small the following holds:

Let $v \in H^{r}\left(D_{0}^{\prime}\right)$ and $v_{h} \in S^{h}(D)$ satisfy $A\left(v-v_{h}, \chi\right)=0$ for all $\chi \in \mathcal{S}^{n}\left(D_{0}^{\prime}\right)$. Then

$$
\left\|v-v_{h}\right\|_{1, D_{1}^{\prime}} \leqslant C\left(h^{r-1}\left\|_{v}\right\|_{r, D_{0}^{\prime}}+\left\|v-v_{h}\right\|_{L_{1}\left(D_{1} \backslash D_{0}\right)}\right) \text {. }
$$

Here $C$ in general depends on the radii of $D_{0}, D_{1}, D$, the quantities $C_{\mathrm{ell}}$ and the constant in (4.1), and $\left\|a_{i j}\right\|_{w_{\infty}^{\alpha}(D)},\left\|b_{i}\right\|_{w_{\infty}^{\beta}(D)},\|d\|_{w_{\infty}^{\gamma}(D)}$ for some fixed $\alpha, \beta, \gamma$.

Proof. We shall first prove $(4.11)$ in the case that $v \equiv 0$ on $D_{0}^{\prime}$, i.e. that if $v_{h}$ satisfies 


$$
A\left(v_{h}, \chi\right)=0 \text { for all } \chi \in \stackrel{C}{S}^{h}\left(D_{0}^{\prime}\right) \text {, }
$$

then

$$
\left\|v_{h}\right\|_{1, D_{1}^{\prime}} \leqslant C\left\|_{v_{h}}\right\|_{L_{1}\left(D_{1} \backslash D_{0}\right)} \text {. }
$$

In order to show this let $D_{0} \subset \subset D_{2} \subset \subset D_{3} \subset \subset D_{4} \subset \subset D_{5} \subset \subset D_{1} \subset \subset D$ be concentric spheres and set $D_{j}^{\prime}=D W_{j}$. Let $\omega \in C^{\infty}(D)$ with $\omega \equiv 1$ on $D_{4}^{\prime}$ and $\omega \equiv$ 0 on $D_{3}$. Then using (4.1), we have

$$
\left\|v_{h}\right\|_{1, D_{1}^{\prime}}^{2} \leqslant\left\|\omega v_{h}\right\|_{1, D}^{2} \leqslant C A\left(\omega v_{h}, \omega v_{h}\right)
$$

A straightforward computation yields that

$$
A\left(\omega v_{h}, \omega v_{h}\right)=A\left(v_{h}, \omega^{2} v_{h}\right)+I,
$$

where

$$
I=\int_{D} v_{h}^{2} \sum_{i, j=1}^{N} a_{i j} D_{i} \omega D_{j} \omega d x+\int_{D} \omega v_{h}^{2} \sum_{i=1}^{N} b_{i} D_{i} \omega d x
$$

Since each $D_{i} \omega$ vanishes outside of $D_{4} D_{3}$, we have that

$$
|I| \leqslant C\left\|_{h}\right\|_{0, D_{4} \backslash D_{3}}^{2} .
$$

Now from (4.12), we have for any $\chi \in \mathcal{S}^{h}\left(D_{0}^{\prime}\right)$,

$$
A\left(v_{h}, \omega^{2} v_{h}\right)=A\left(v_{h}, \omega^{2} v_{h}-\chi\right) .
$$

By A.3 we can find an $\eta \in \xi^{n}\left(D_{5}\right)$ with $\eta \equiv v_{n}$ on $D_{2}$ such that

$$
\left\|\left(1-\omega^{2}\right) v_{h}-\eta\right\|_{1, D} \leqslant C h\left\|_{h}\right\|_{1, D_{5} \backslash D_{2}} .
$$

Choosing $\chi$ in (4.17) as $\chi=v_{h}-\eta$, we have that $\omega^{2} v_{h}-\chi$ vanishes outside of $D_{5} \backslash D_{2}$ and

$$
\left\|\omega^{2} v_{h}-\chi\right\|_{1, D_{5} \backslash D_{2}} \leqslant\left\|\left(1-\omega^{2}\right) v_{h}-\eta\right\|_{1, D} .
$$

Hence from (4.17) and (4.18),

$$
A\left(v_{h}, \omega^{2} v_{h}\right) \leqslant C h\left\|_{n}\right\|_{1, D_{5} \backslash D_{2}}^{2}
$$

From (4.14), (4.15), (4.16) and (4.19) we obtain

$$
\left\|v_{h}\right\|_{1, D_{1}^{\prime}} \leqslant C\left\|_{v_{h}}\right\|_{1, D_{5} \backslash D_{2}},
$$

and the desired result (4.13) follows on applying (3.4) of Lemma 3.1 to the righthand side of (4.20).

We are now in position to prove (4.11). With $\omega$ as before, let $(\omega v)_{n} \in S^{h}(D)$ satisfy $A\left(\omega v-(\omega v)_{h}, \chi\right)=0$ for all $\chi \in S^{h}(D)$. Then

$$
\left\|v-v_{h}\right\|_{1, D_{1}^{\prime}} \leqslant\left\|\omega v-(\omega v)_{h}\right\|_{1, D_{1}^{\prime}}+\left\|(\omega v)_{h}-v_{h}\right\|_{1, D_{1}^{\prime}}
$$

From Lemma 4.3 we obtain 


$$
\left\|\omega v-(\omega v)_{h}\right\|_{1, D} \leqslant C h^{r-1}\|\omega v\|_{r, D} \leqslant C h^{r-1}\left\|_{v}\right\|_{r, D}^{\prime} .
$$

Since $A\left((\omega v)_{h}-v_{h}, \chi\right)=0$ for all $\chi \in \mathcal{S}^{h}\left(D_{5}^{\prime}\right)$, it follows from (4.13) (with $D_{0}$ replaced by $D_{5}$ ), the triangle inequality and (4.22) that

$$
\begin{aligned}
& \left\|(\omega v)_{h}-v_{h}\right\|_{1, D_{1}^{\prime}} \leqslant C\left\|(\omega v)_{h}-v_{h}\right\|_{L_{1}\left(D_{1} \backslash D_{5}\right)} \\
& \leqslant C\left(\left\|\omega v-(\omega v)_{h}\right\|_{L_{1}\left(D_{1} \backslash D_{5}\right)}+\left\|v-v_{h}\right\|_{L_{1}\left(D_{1} \backslash D_{5}\right)}\right) \\
& \leqslant C\left(h^{r-1}\|v\|_{r, D_{0}^{\prime}}+\left\|v-v_{h}\right\|_{L_{1}\left(D_{1} \backslash D_{5}\right)}\right) .
\end{aligned}
$$

The inequality (4.11) now follows from (4.21), (4.22) and (4.23). This completes the proof of the lemma.

5. Interior Maximum Norm Estimates. In this section we shall state the main result of this paper. We shall prove it here for coercive forms. The case of noncoercive forms will be treated in Appendix 1.

Theorem 5.1. Assume that A.0, A.1, A.2, A.3, A.4 and (3.1) hold. Let $\Omega_{1} \subset \subset$ $\Omega$, let $p$ be a nonnegative integer, and $1 \leqslant q \leqslant \infty$. There exists a constant $C$ and an $0<h_{1}<1$ such that the following holds:

Let $u$ be continuous on $\Omega$ and $u_{n} \in S^{n}(\Omega)$ satisfy

$$
A\left(u-u_{h}, \chi\right)=0 \text { for all } \chi \in \xi^{h}(\Omega) \text {. }
$$

Then for $0<h \leqslant h_{1}$ and any $\chi \in S^{h}(\Omega)$,

$$
\left\|u-u_{h}\right\|_{L_{\infty}\left(\Omega_{1}\right)} \leqslant C\left\{(\ln 1 / h)^{\bar{r}}\left\|_{u}-\chi\right\|_{L_{\infty}(\Omega)}+\left\|u-u_{h}\right\|_{w_{q}^{-p}(\Omega)}\right\}
$$

where

$$
\bar{r}= \begin{cases}1 & \text { if } r=2, \\ 0 & \text { if } r \geqslant 3\end{cases}
$$

Proof of Theorem 5.1 for Coercive Forms $A$. We shall make the additional hypothesis that the form $A$ is coercive, i.e., satisfies (4.1). The case of noncoercive forms will be given in Appendix 1. In what follows, $D \subset \subset \Omega$ will be a sphere of radius $R$ $>0$ with center at $x_{0}$, where $x_{0} \in \bar{\Omega}_{1}$ is such that $\left\|u-u_{h}\right\|_{L_{\infty}\left(\Omega_{1}\right)}=\left|\left(u-u_{h}\right)\left(x_{0}\right)\right|$. We shall need the following lemmas.

LEMмA 5.1. Under the above hypotheses, there exists a constant $C$ such that the following holds:

Let $\tilde{u}$ have compact support in the sphere $1 / 2 D$. If $\tilde{u}_{h} \in S^{h}(D)$ satisfies

$$
A\left(\tilde{u}-\tilde{u}_{h}, \chi\right)=0 \text { for all } \chi \in S^{h}(D),
$$

then for $0<h \leqslant h_{1}$,

$$
\left\|\tilde{u}-\tilde{u}_{h}\right\|_{L_{\infty}(1 / 4 D)} \leqslant C(\ln 1 / h)^{\bar{r}}\|\tilde{u}\|_{L_{\infty}(D)} .
$$

LEMmA 5.2. Under the above hypotheses, there exists a constant $C$ such that if $w_{h} \in S^{h}(D)$ satisfies 


$$
A\left(w_{h}, \chi\right)=0 \text { for all } \chi \in S^{h}(D) \text {, }
$$

then

$$
\left|w_{h}\left(x_{0}\right)\right| \leqslant C\left\|w_{h}\right\|_{w_{q}^{-p}(D)} .
$$

Before proving Lemmas 5.1 and 5.2, let us show how Theorem 5.1 follows in the coercive case. Let $\omega \in \mathcal{C}^{\infty}(1 / 2 D)$ with $\omega \equiv 1$ on $1 / 4 D$ and set $\tilde{u}=\omega u$. Then taking $\tilde{u}_{n} \in S^{h}(D)$ satisfying (5.2), we have from (5.3) that

$$
\left|u\left(x_{0}\right)-\tilde{u}_{h}\left(x_{0}\right)\right| \leqslant C(\ln 1 / h)^{\bar{r}}\|\tilde{u}\|_{L_{\infty}(D)} \leqslant C(\ln 1 / h)^{\bar{r}}\|u\|_{L_{\infty}(D)} .
$$

For $\eta \in S^{n}(1 / 4 D)$ we obtain

$$
A\left(\tilde{u}_{h}-u_{h}, \eta\right)=A\left(u-u_{n}, \eta\right)=0 .
$$

Hence taking $w_{h}=\tilde{u}_{h}-u_{h}$, it follows from (5.5) (with $D$ replaced by $1 / 4 D$ ) and (5.3) that

$$
\begin{aligned}
\left|\left(\tilde{u}_{h}-u_{h}\right)\left(x_{0}\right)\right| & \leqslant C\left\|\tilde{u}_{h}-u_{h}\right\|_{w_{q}^{-p}(1 / 4 D)} \\
& \leqslant C\left\{\left\|\tilde{u}-\tilde{u}_{h}\right\|_{L_{\infty}(1 / 4 D)}+\left\|u-u_{h}\right\|_{w_{q}^{-p}(1 / 4 D)}\right\} \\
& \leqslant C\left\{(\ln 1 / h)^{\bar{r}}\left\|_{u}\right\|_{L_{\infty}(D)}+\left\|u-u_{h}\right\|_{w_{q}^{-p}(D)}\right\} .
\end{aligned}
$$

Using this and (5.6), we obtain via the triangle inequality

$$
\left\|u-u_{h}\right\|_{L_{\infty}\left(\Omega_{1}\right)} \leqslant C\left\{(\ln 1 / h)^{\bar{r}}\|u\|_{L_{\infty}(\Omega)}+\left\|u-u_{h}\right\|_{w_{q}^{-p}(\Omega)}\right\}
$$

and the theorem follows upon writing $u-u_{n}=(u-\chi)-\left(u_{n}-\chi\right)$ for $\chi \in S^{h}(\Omega)$.

The proofs of Lemmas 5.1 and 5.2 will depend on the following technical lemma.

LEMmA 5.3. Under the hypotheses of Theorem 5.1 , let $D_{h} \subset \subset 1 / 4 D$ be a sphere of radius $C^{\prime} h$. There exists a constant $C$ such that for $0<h \leqslant h_{1}$ the following holds.

Let $\varphi \in \mathcal{C}^{\infty}\left(D_{h}\right)$, and let $v$ and $v_{h} \in S^{h}(D)$ satisfy

$$
\begin{aligned}
A(\psi, v) & =(\psi, \varphi) & & \text { for all } \psi \in H^{1}(D), \\
A\left(\chi, v-v_{h}\right) & =0 & & \text { for all } \chi \in S^{h}(D) .
\end{aligned}
$$

Then

and for $D_{1} \subset \subset D$,

$$
\left\|v-v_{h}\right\|_{w_{1}^{1}(D)} \leqslant C h^{N / 2+1}(\ln 1 / h)^{\bar{r}}\left\|_{\varphi}\right\|_{0, D_{h}}
$$

$$
\left\|v-v_{h}\right\|_{w_{1}^{2, h}\left(D_{1}\right)} \leqslant C h^{N / 2}(\ln 1 / h)^{\bar{r}}\left\|_{\varphi}\right\|_{0, D_{h}} .
$$

Assuming Lemma 5.3 for the moment, we shall prove Lemmas 5.1 and 5.2.

Proof of Lemma 5.1. Let $\left\|\tilde{u}-\tilde{u}_{n}\right\|_{L_{\infty}(1 / 4 D)}=\left|\left(\tilde{u}-\tilde{u}_{n}\right)\left(x_{1}\right)\right|$. For simplicity in notation we shall assume that $x_{1}$ is the center of $D$. Let $D_{h}$ be a sphere of radius $C^{\prime} h, C^{\prime}>k$, with center at $x_{1}$. Then 


$$
\begin{aligned}
\left|\left(\tilde{u}-\tilde{u}_{h}\right)\left(x_{1}\right)\right| & \leqslant\left|\tilde{u}\left(x_{1}\right)\right|+\left|\tilde{u}_{h}\left(x_{1}\right)\right| \\
& \leqslant\left|\tilde{u}\left(x_{1}\right)\right|+C h^{-N / 2}\left\|\tilde{u}_{h}\right\|_{L_{2}\left(D_{h}\right)} \\
& \leqslant\left|\tilde{u}\left(x_{1}\right)\right|+C h^{-N / 2}\left\|\tilde{u}_{h}-\tilde{u}\right\|_{L_{2}\left(D_{h}\right)}+C h^{-N / 2}\|\tilde{u}\|_{L_{2}\left(D_{h}\right)} \\
& \leqslant C\left\{\|\tilde{u}\|_{L_{\infty}\left(D_{h}\right)}+h^{-N / 2}\left\|\tilde{u}_{h}-\tilde{u}\right\|_{L_{2}\left(D_{h}\right)}\right\} .
\end{aligned}
$$

Here we used (2.2.b) and the triangle inequality.

Now (5.3) would follow once we had shown that

$$
h^{-N / 2}\left\|\tilde{u}-\tilde{u}_{h}\right\|_{0, D_{h}} \leqslant C(\ln 1 / h)^{\bar{r}}\|\tilde{u}\|_{L_{\infty}(D)} .
$$

We have

$$
\left\|\tilde{u}-\tilde{u}_{h}\right\|_{0, D_{h}}=\sup _{\varphi \in \tilde{C}^{\infty}\left(D_{h}\right)} \frac{\left(\tilde{u}-\tilde{u}_{h}, \varphi\right)}{\|\varphi\|_{0, D_{h}}} .
$$

For each such $\varphi$, let $v$ and $v_{h}$ satisfy (5.7) and (5.8). Then

$$
\left(\tilde{u}-\tilde{u}_{h}, \varphi\right)=A\left(\tilde{u}-\tilde{u}_{h}, v\right)=A\left(\tilde{u}, v-v_{h}\right) .
$$

Let $L$ denote the second order operator corresponding to $A$ (given by $(0.1)$ ), $L^{*}$ its formal adjoint, and $\partial / \partial n$ the conormal derivative. Integrating by parts, we have

$$
A\left(\tilde{u}, v-v_{h}\right)=\sum_{i \in I} \int_{\tau_{i}^{n}} \tilde{u} L^{*}\left(v-v_{h}\right) d x+\sum_{i \in I} \int_{\partial \tau_{i}^{n}} \tilde{u} \frac{\partial}{\partial n}\left(v-v_{h}\right) d \sigma,
$$

where, since $\tilde{u}$ vanishes outside $1 / 2 D$, we may take $I=\left\{i \mid \tau_{i}^{h} \cap 1 / 2 D \neq \varnothing\right\}$. Let $D_{I}=$ $\bigcup_{i \in I} \bar{\tau}_{i}^{h}$. Then

$$
\left|\sum_{i \in I} \int_{\tau_{i}^{h}} \tilde{u} L^{*}\left(v-v_{h}\right) d x\right| \leqslant C\|\tilde{u}\|_{L_{\infty}(D)}\left\|_{v}-v_{h}\right\|_{w_{1}^{2, h}\left(D_{I}\right)} .
$$

In view of our assumption A.0,

$$
\left|\sum_{i \in I} \int_{\partial \tau_{i}^{n}} \tilde{u} \frac{\partial\left(v-v_{h}\right)}{\partial n} d \sigma\right| \leqslant C\|\tilde{u}\|_{L_{\infty}(D)}\left(h^{-1}\left\|_{v}-v_{h}\right\|_{w_{1}^{1}\left(D_{I}\right)}+\left\|v-v_{h}\right\|_{w_{1}^{2, h}\left(D_{I}\right)}\right) \text {, }
$$

and therefore,

$$
\left|A\left(\tilde{u}, v-v_{h}\right)\right| \leqslant C\|\tilde{u}\|_{L_{\infty}(D)}\left(h^{-1}\left\|_{v}-v_{h}\right\|_{w_{1}^{1}\left(D_{I}\right)}+\left\|v-v_{h}\right\|_{w_{1}^{2, h}\left(D_{I}\right)}\right) .
$$

Now using Lemma 5.3 we obtain

$$
\left|A\left(\tilde{u}, v-v_{h}\right)\right| \leqslant C\|\tilde{u}\|_{L_{\infty}(D)} h^{N / 2}(\ln 1 / h)^{\bar{r}}\left\|_{\varphi}\right\|_{0, D_{h}},
$$

and (5.11) follows from this, (5.13) and (5.12). This proves Lemma 5.1.

Proof of Lemma 5.2. Using Proposition 2.2, there exists a function $\eta_{h} \in$ $S^{h}(3 / 4 D)$ such that $\eta_{h} \equiv w_{h}$ on $1 / 2 D$ and $\left\|\eta_{h}\right\|_{1,3 / 4 D} \leqslant C\left\|_{h}\right\|_{1, D}$. Then

$$
\left|w_{h}\left(x_{0}\right)\right|=\left|\eta_{h}\left(x_{0}\right)\right| \leqslant C h^{-N / 2}\left\|\eta_{h}\right\|_{0, D_{h}}=C h^{-N / 2} \sup _{\varphi \in C^{\infty}\left(D_{h}\right)} \frac{\left(\eta_{h}, \varphi\right)}{\|\varphi\|_{0, D_{h}}},
$$

where diam $D_{n} \leqslant C h$. Let $\varphi \in \mathcal{C}^{\infty}\left(D_{h}\right)$ be fixed and let $v$ and $v_{n}$ satisfy (5.7) and (5.8) so that 


$$
\left(\eta_{h}, \varphi\right)=A\left(\eta_{h}, v\right)=A\left(\eta_{h}, v_{h}\right) .
$$

Let $\chi_{h} \in S^{h}(1 / 2 D)$ with $\chi_{h} \equiv v_{h}$ on $1 / 4 D$ be as $\eta$ in Proposition 2.2. Since $A\left(\eta_{h}, \chi_{h}\right)=$ $A\left(w_{h}, \chi_{h}\right)=0$, and using (2.5), we have

$$
\begin{aligned}
\left(\eta_{h}, \varphi\right) & =A\left(\eta_{h}, v_{h}-\chi_{h}\right) \leqslant C\left\|_{n}\right\|_{1,3 D / 4}\left\|v_{h}\right\|_{1,3 D / 4 \backslash D / 8} \\
& \leqslant C\left\|w_{h}\right\|_{1, D}\left\|v_{h}\right\|_{1,3 D / 4 \backslash D / 8} .
\end{aligned}
$$

Since $v_{h}$ satisfies $A\left(\chi, v_{h}\right)=0$ for $\chi \in S^{h}\left(D \backslash D_{h}\right)$, (3.4) yields

$$
\left\|v_{h}\right\|_{1,3 D / 4 \backslash D / 8} \leqslant C\left\|_{v_{n}}\right\|_{w_{1}^{1}(D)} .
$$

Using Lemma 5.3 and (4.8),

$$
\left\|v_{h}\right\|_{w_{1}^{1}(D)} \leqslant\left\|v-v_{h}\right\|_{w_{1}^{1}(D)}+\|v\|_{w_{1}^{1}(D)} \leqslant C h^{N / 2}\|\varphi\|_{0, D_{h}} .
$$

Thus, from (5.16), (5.15) and (5.14) we obtain

$$
\left|w_{h}\left(x_{0}\right)\right| \leqslant C\left\|w_{h}\right\|_{1, D} .
$$

Replacing $D$ by $1 / 2 D$, the desired result (5.5) follows from (3.4). This proves Lemma 5.2.

Proof of Lemma 5.3. We shall first prove (5.9). For simplicity in notation, let $D_{h}$ and $D$ be concentric spheres with centers at the origin and with radii $h$ and 1 , respectively. Let $\Omega_{j}$ denote the annuli

$$
\Omega_{j}=\left\{x\left|2^{-j-1} \leqslant\right| x \mid \leqslant 2^{-j}\right\},
$$

and let $J$ be the largest integer such that $2^{-J} \geqslant C_{*} h$, where $C_{*}$ is to be chosen later (sufficiently large). Set $d_{j}=2^{-j}, \Omega_{h}=D \backslash \bigcup_{j=0}^{J} \Omega_{j}$, and let

$$
\Omega_{j}^{l}=\left(\Omega_{j-l} \cup \bar{\Omega}_{j-l+1} \cup \cdots \cup \Omega_{j+l}\right) \cap D, \quad l=1,2, \ldots
$$

Furthermore, set $e=v-v_{h}$. We have

$$
\|e\|_{w_{1}^{1}(D)}=\sum_{j=0}^{J}\|e\|_{w_{1}^{1}\left(\Omega_{j}\right)}+\|e\|_{w_{1}^{1}\left(\Omega_{h}\right)} .
$$

Using Lemmas 4.3 and 4.1 , we obtain

$$
\|e\|_{w_{1}^{1}\left(\Omega_{h}\right)} \leqslant C C_{*}^{N / 2} h^{N / 2}\left\|_{e}\right\|_{1, \Omega_{h}} \leqslant C C_{*}^{N / 2} h^{N / 2+1}\left\|_{\varphi}\right\|_{0, D_{h}} .
$$

By Lemma 3.2, or Lemma 4.4 in the case of $j=0$, it follows that

$$
\|e\|_{w_{1}^{1}\left(\Omega_{j}\right)} \leqslant C d_{j}^{N / 2}\|e\|_{1, \Omega_{j}} \leqslant C d_{j}^{N / 2}\left\{h^{r-1}\left\|_{v \|_{r, \Omega_{j}^{1}}}+d_{j}^{-N / 2-1}\right\| e \|_{L_{1}\left(\Omega_{j}^{1}\right)}\right\} .
$$

By (4.3) we have

and thus

$$
\|v\|_{r, \Omega_{j}^{1}} \leqslant C d_{j}^{-N / 2+2-r} h^{N / 2}\|\varphi\|_{0, D_{h}}
$$




$$
\begin{aligned}
\sum_{j=0}^{J}\|e\|_{w_{1}^{1}\left(\Omega_{j}\right)} & \leqslant C\left(\sum_{j=0}^{J} d_{j}^{2-r} h^{N / 2+r-1}\right)\|\varphi\|_{0, D_{h}}+C \sum_{j=0}^{J} d_{j}^{-1}\|e\|_{L_{1}\left(\Omega_{j}^{1}\right)} \\
& \leqslant C h^{N / 2+1}(\ln 1 / h)^{\bar{r}}\left\|_{\varphi}\right\|_{0, D_{h}}+C \cdot I
\end{aligned}
$$

where

$$
I=\sum_{j=0}^{J} d_{j}^{-1}\|e\|_{L_{1}\left(\Omega_{j}^{1}\right)} .
$$

We shall now estimate $I$. Let

$$
\tilde{I}=\frac{1}{C_{*} h}\|e\|_{L_{1}\left(\Omega_{h}\right)}+\sum_{j=0}^{J} d_{j}^{-1}\|e\|_{L_{1}\left(\Omega_{j}\right)}
$$

and note that $I \leqslant 8 \tilde{I}$. Now,

$$
\frac{1}{C_{*} h}\|e\|_{L_{1}\left(\Omega_{h}\right)} \leqslant C\left(C_{*}\right)^{N / 2-1} h^{N / 2-1}\|e\|_{L_{2}\left(\Omega_{h}\right)}
$$

and by Lemma 4.3 and 4.1 ,

$$
\frac{1}{C_{*} h}\|e\|_{L_{1}\left(\Omega_{h}\right)} \leqslant C\left(C_{*}\right)^{N / 2-1} h^{N / 2+1}\|\varphi\|_{0, D_{h}} .
$$

Next write

$$
\|e\|_{L_{1}\left(\Omega_{j}\right)}=\sup _{\eta \in C^{\circ \infty}\left(\Omega_{j}\right)} \frac{(e, \eta)}{\|\eta\|_{L_{\infty}\left(\Omega_{j}\right)}} .
$$

Letting $A(w, \psi)=(w, \eta)$ for $w \in H^{1}(D)$ and letting $\chi \in S^{h}(D)$,

$$
\|e\|_{L_{1}\left(\Omega_{j}\right)}=\sup _{\eta \in C^{\infty}\left(\Omega_{j}\right)} \frac{A(e, \psi-\chi)}{\|\eta\|_{L_{\infty}\left(\Omega_{j}\right)}} .
$$

We have

(5.22) $A(e, \psi-\chi) \leqslant\|e\|_{w_{1}^{1}\left(D \backslash \Omega_{j}^{2}\right)}\|\psi-\chi\|_{w_{\infty}^{1}\left(D \backslash \Omega_{j}^{2}\right)}+\|e\|_{1, \Omega_{j}^{2}}\|\psi-\chi\|_{1, \Omega_{j}^{2}}$.

By A.1 (cf. Proposition 2.2 for $j=0$ ) and by (4.7) we obtain for a suitable $\chi$,

$$
\|\psi-\chi\|_{w_{\infty}^{1}\left(D \backslash \Omega_{j}^{2}\right)} \leqslant C h\|\psi\|_{w_{\infty}^{2}\left(D \backslash \Omega_{j}^{1}\right)} \leqslant C h\|\eta\|_{L_{\infty}\left(\Omega_{j}\right)} .
$$

Furthermore, by Lemma 3.2 and (4.3),

$$
\begin{aligned}
& \|e\|_{1, \Omega_{j}^{2}} \leqslant C h\left\|_{v}\right\|_{2, \Omega_{j}^{3}}+C d_{j}^{-N / 2-1}\|e\|_{L_{1}\left(\Omega_{j}^{3}\right)} \\
& \leqslant C h d_{j}^{-N / 2} h^{N / 2}\|\varphi\|_{0, D_{h}}+C d_{j}^{-N / 2-1}\|e\|_{L_{1}\left(\Omega_{j}^{3}\right)} ;
\end{aligned}
$$

and we can take $\chi$ such that

$$
\|\psi-\chi\|_{1, \Omega_{j}^{2}} \leqslant C h\|\psi\|_{2, \Omega} \leqslant C h\left\|_{\eta \|_{0, \Omega_{j}}} \leqslant C h d_{j}^{N / 2}\right\| \eta \|_{L_{\infty}\left(\Omega_{j}\right)} .
$$

Thus, 


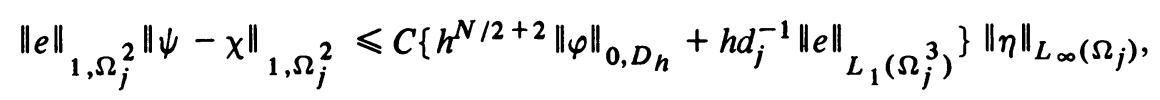

and from this and (5.21), (5.22) and (5.23),

$$
\|e\|_{L_{1}\left(\Omega_{j}\right)} \leqslant C\left\{h\left\|_{w_{1}\left(D \backslash \Omega_{j}^{2}\right)}+h d_{j}^{-1}\right\| e\left\|_{L_{1}\left(\Omega_{j}^{3}\right)}+h^{N / 2+2}\right\|_{\varphi} \|_{0, D_{h}}\right\} .
$$

Now using (5.20), we have

$$
\begin{aligned}
& \tilde{I} \leqslant C\left\{C_{*}^{N / 2-1} h^{N / 2+1}+h^{N / 2+2} \sum_{j=0}^{J} d_{j}^{-1}\right\}\|\varphi\|_{0, D_{h}} \\
& +C h \sum_{j=0}^{J}\left(d_{j}^{-1}\|e\|_{w_{1}^{1}\left(D \backslash \Omega_{j}^{2}\right)}\right)+C \sum_{j=0}^{J}\left(\frac{h}{d_{j}}\right) d_{j}^{-1}\|e\|_{L_{1}\left(\Omega_{j}^{3}\right)} \\
& \leqslant C C_{*}^{N / 2-1} h^{N / 2+1}\|\varphi\|_{0, D_{h}}+\frac{C}{C_{*}}\|e\|_{w_{1}^{1}(D)}+\frac{C}{C_{*}} \tilde{I}
\end{aligned}
$$

Thus, choosing $C_{*}$ large enough we obtain

$$
\tilde{I} \leqslant C C_{*}^{N / 2-1} h^{N / 2+1}\left\|_{\varphi}\right\|_{0, D_{h}}+\frac{C}{C_{*}}\|e\|_{w_{1}^{1}(D)},
$$

and from this, (5.17), (5.18) and (5.19),

Hence,

$$
\|e\|_{w_{1}^{1}(D)} \leqslant C C_{*}^{N / 2} h^{N / 2+1}(\ln 1 / h)^{\bar{r}}\left\|_{\varphi}\right\|_{0, D_{h}}+\frac{C}{C_{*}}\|e\|_{w_{1}^{1}(D)} .
$$

$$
\|e\|_{w_{1}^{1}(D)} \leqslant C h^{N / 2+1}(\ln 1 / h)^{\bar{r}}\left\|_{\varphi}\right\|_{0, D_{h}},
$$

which completes the proof of (5.9).

We shall now prove (5.10). Let $D_{n} \subset \subset D_{2 n} \subset \subset D_{3 n} \subset \subset D_{4 n} \subset \subset D_{5 n}$ $\subset \subset D_{6 h}$ be concentric spheres with $\operatorname{dist}\left(D_{j h}, \partial D_{(j+1) h}\right)=k h, j=1, \ldots, 5$. We have

$$
\left\|v-v_{h}\right\|_{w_{1}^{2, h}\left(D_{1}\right)} \leqslant\left\|v-v_{h}\right\|_{w_{1}^{2, h}\left(D_{1} \backslash D_{4 h}\right)}+\left\|v-v_{h}\right\|_{w_{1}^{2, n}\left(D_{4 h}\right)} .
$$

For $\chi \in S^{h}(D)$ it follows from (2.2.b) that

$$
\begin{aligned}
& \left\|v-v_{h}\right\|_{w_{1}^{2, h}\left(D_{4 h}\right)} \leqslant\left\|_{v}-\chi\right\|_{w_{1}^{2, h}\left(D_{4 h}\right)}+C h^{-1}\left\|_{\chi}-v_{h}\right\|_{w_{1}^{1}\left(D_{5 h}\right)} \\
& \leqslant\|v-\chi\|_{w_{1}^{2, h}\left(D_{4 h}\right)}+C h^{-1}\left\|_{v}-\chi\right\|_{w_{1}^{1}\left(D_{5 h}\right)}+C h^{-1}\left\|_{v}-v_{h}\right\|_{w_{1}^{1}(D)}
\end{aligned}
$$

Choosing $\chi$ to satisfy (2.1), we have

$$
\begin{aligned}
& \|v-\chi\|_{w_{1}^{2, h}\left(D_{4 h}\right)}+C h^{-1}\left\|_{v}-\chi\right\|_{w_{1}^{1}\left(D_{5 h}\right)} \\
& \leqslant C\left\|_{v}\right\|_{w_{1}^{2}\left(D_{6 h}\right)} \leqslant C h^{N / 2}\left\|_{v \|_{2, D_{6 h}}} \leqslant C h^{N / 2}\right\| \varphi \|_{0, D_{h}} .
\end{aligned}
$$

From this, (5.25) and (5.9) we obtain 


$$
\left\|v-v_{h}\right\|_{w_{1}^{2, h}\left(D_{4 h}\right)} \leqslant C h^{N / 2}(\ln 1 / h)^{\bar{r}}\|\varphi\|_{0, D_{h}} .
$$

As in (5.25), we have that for any $\eta \in S^{h}(D)$

$$
\begin{aligned}
& \left\|v-v_{h}\right\|_{w_{1}^{2, h}\left(D \backslash D_{4 h}\right)} \\
& \quad \leqslant\|v-\eta\|_{w_{1}^{2, h}\left(D \backslash D_{4 h}\right)}+C h^{-1}\left\|_{v}-\eta\right\|_{w_{1}^{1}\left(D \backslash D_{3 h}\right)}+C h^{-1}\left\|_{v}-v_{h}\right\|_{w_{1}^{1}(D)} .
\end{aligned}
$$

Choosing $\eta$ to satisfy (2.1) and using (4.4), we arrive at

$$
\begin{aligned}
& \|v-\eta\|_{w_{1}^{2, h}\left(D \backslash D_{4 h}\right)}+C h^{-1}\|v-\eta\|_{w_{1}^{1}\left(D \backslash D_{3 h}\right)} \\
& \leqslant C h^{r-2}\|v\|_{w_{1}^{r}\left(D \backslash D_{2 h}\right)} \leqslant C\left(\ln \frac{1}{h}\right)^{\bar{r}}\left\|_{\varphi}\right\|_{L_{1}\left(D_{h}\right)} \leqslant C h^{N / 2}\left(\ln \frac{1}{h}\right)^{\bar{r}}\|\varphi\|_{0, D_{h}} .
\end{aligned}
$$

This together with (5.9), (5.24), (5.26) and (5.27) proves (5.10).

This completes the proof of Lemma 5.3.

The proof of Theorem 5.1 in the case that the form $A$ is coercive is now accomplished. As mentioned previously, the case of noncoercive $A$ will be given in Appendix 1.

Theorem 5.1 was proved under the assumption that the domains $\Omega_{1}$ and $\Omega$ were fixed. Analogously to Lemma 3.2 we have the following result when the domains may vary and be close with $h$.

COROllary 5.1. Assume that the conditions of Theorem 5.1 hold and let $\Omega_{1} \subset \subset \Omega_{2} \subset \subset \Omega$. There exist constants $C_{6}>0$ and $C$ such that if $C_{6} h \leqslant d$, $\operatorname{dist}\left(\Omega_{1}, \partial \Omega_{2}\right) \geqslant d$, and $\operatorname{dist}\left(\Omega_{2}, \partial \Omega\right) \geqslant d$, then for $0 \leqslant l \leqslant r$,

$$
\left\|u-u_{h}\right\|_{L_{\infty}\left(\Omega_{1}\right)} \leqslant C\left\{h^{l}\left(\ln \frac{d}{h}\right)^{\bar{r}}|u|_{w_{\infty}^{l}\left(\Omega_{2}\right)}+d^{-N / q-p}\left\|u-u_{h}\right\|_{w_{q}^{-p}\left(\Omega_{2}\right)}\right\},
$$

where $C$ is independent of $h, u, u_{h}, \Omega_{1}, \Omega_{2}$ and in general depends on $p, C_{\mathrm{ell}}$, $\left\|a_{i j}\right\|_{w_{\infty}^{\alpha}(\Omega)},\left\|b_{i}\right\|_{w_{\infty}^{\beta}(\Omega)},\|d\|_{w_{\infty}^{\gamma}(\Omega)}$ for some $\alpha, \beta, \gamma$.

Proof. We shall mimic the proof of Lemma 3.2. Let $x_{0} \in \bar{\Omega}_{1}$ be such that $\left|\left(u-u_{h}\right)\left(x_{0}\right)\right|=\left\|u-u_{h}\right\|_{L_{\infty}\left(\Omega_{1}\right)}$. Without loss of generality we assume that $\Omega_{1}$ and $\Omega_{2}$ are concentric spheres with center at $x_{0}$ and diam $\Omega_{1}=2 d$. Let $y=$ $\left(x-x_{0}\right) / d$ and transform the problem to the new variables $y$ on $\widetilde{\Omega}_{2}$. The new sets $\tilde{\tau}_{i}^{h}$ satisfy A.0 with $h$ replaced by $h / d$. From A.4 it follows that A.1, A.2 and A.3 are satisfied on the new domain $\widetilde{\Omega}_{2}$ with $h$ replaced by $h / d$. To verify that A.4 is satisfied on $\widetilde{\Omega}_{2}$, let $y_{0} \in \widetilde{\Omega}_{2}$ and transform a sphere of radius $\widetilde{d} \geqslant C_{4} h / d$ in $\widetilde{\Omega}_{2}$ via the transformation $z=\left(y-y_{0}\right) / \widetilde{d}$. It is seen that this is equivalent to transforming a sphere of radius $d \widetilde{d}$ in $\Omega_{2}$ via the transformation $z=\left(x-x_{1}\right) / d \widetilde{d}$ for some point $x_{1} \in \Omega_{2}$. Since $d \widetilde{d} \geqslant C_{4} h$, A.4 yields that A.1, A.2, and A.3 also hold with $h / d$ replaced by $h / d \widetilde{d}$. Hence Corollary 5.1 is valid on the domains $\widetilde{\Omega}_{1}$ and $\widetilde{\Omega}_{2}$, and the desired result follows by transforming back to the original domains.

6. Pointwise Error Estimates for the Green's Function Near the Singularity. In this section we shall apply the results of Section 5 to derive estimates for the error in 
the Green's function. We shall restrict our attention to the Neumann problem on a smooth domain, but the techniques given below are suitable for investigating other boundary value problems and methods for solving them.

Let $D$ be a bounded domain in $R^{N}$ with a smooth boundary $\partial D$, and let $A$ be a bilinear form of the type (0.4), where $A$ is coercive over $H^{1}$, i.e., there exists a constant $c>0$ such that

$$
c\|v\|_{1, D}^{2} \leqslant A(v, v) \text { for all } v \in H^{1}(D) .
$$

Let $S^{h}(D)$ be a one parameter family of subspaces of $W_{\infty}^{1}(D)$ having the global property that

$$
\inf _{x \in S^{h}(0)}\|v-\chi\|_{1, D} \leqslant C h^{l-1}\|v\|_{l, D} \text { for } 1 \leqslant l \leqslant r .
$$

In addition, the spaces will be required to satisfy the conditions of Theorem 5.1 on interior subdomains.

Let $y \in D$ and $G^{(y)}(x)$ and $G_{h}^{(y)}(x) \in S^{h}(D)$, respectively, be the Green's function and approximate Green's function defined by

$$
A\left(G^{(y)}, v\right)=v(y) \quad \text { for all } v \in W_{\infty}^{1}(D),
$$

and

$$
A\left(G_{h}^{(y)}, \chi\right)=\chi(y) \text { for all } \chi \in S^{h}(D) .
$$

We wish to estimate $\left|G^{(y)}(x)-G_{h}^{(y)}(x)\right|$ where $y$ is in the interior of $D$ and $x$ may be close to $y$. More precisely we have:

TheOrem 6.1. Assume (6.1) and (6.2). Let $\Omega_{1} \subset \subset \Omega_{2} \subset \subset D$ and suppose that the conditions of Corollary 5.1 are satisfied. There exist constants $C$ and $C_{7}$ such that if $h$ is sufficiently small, then for $y \in \Omega_{1}, x \in \Omega_{2}$.

(i) If $|x-y| \geqslant C_{7} h$,

(ii) If $0<|x-y| \leqslant C_{7} h$,

$$
\left|G^{(y)}(x)-G_{h}^{(y)}(x)\right| \leqslant C h^{r}\left(\ln \left(\frac{|x-y|}{h}\right)\right)^{\bar{r}}|| x-\left.y\right|^{N-2+r} .
$$

$$
\left|G^{(y)}(x)-G_{h}^{(y)}(x)\right| \leqslant C \begin{cases}\ln \frac{1}{|x-y|}+1 & \text { for } N=2, \\ \frac{1}{|x-y|^{N-2}} & \text { for } N \geqslant 3 .\end{cases}
$$

This theorem can be applied, e.g. in the following situation, cf. [5]. Let $f$ have compact support in $D$. Then

$$
\left(u-u_{h}\right)(y)=\int_{\operatorname{supp~} f}\left(G^{(y)}(x)-G_{h}^{(y)}(x)\right) f(x) d x,
$$

and using the results (6.3) and (6.4), the error can be estimated in various domains under weak regularity assumptions on $f$.

Proof. Let $d=|x-y|$ and denote by $B_{r}(x)$ the sphere of radius $r$ around $x$. Furthermore, set $e^{(y)}=G^{(y)}-G_{h}^{(y)}$. Note that there exist a fixed domain $\Omega_{3}$ 
independent of $d, \Omega_{2} \subset \subset \Omega_{3} \subset \subset D$, and a constant $c_{1}, 0<c_{1} \leqslant 1 / 2$ such that for any $x \in \Omega_{2}$,

$$
D \equiv B_{c_{1} d}(x) \subset \subset \Omega_{3}
$$

Let $D_{1}=B_{1 / 2 c_{1} d}(x) \subset \subset D$. Applying Corollary 5.1 to $D_{1} \subset \subset D$, we obtain

$$
\left|e^{(y)}(x)\right| \leqslant C\left\{h^{r}\left(\ln \frac{d}{h}\right)^{\bar{r}}\left|G^{(y)}\right|_{w_{\infty}^{r}(D)}+d^{-N-r+2}\left\|e^{(y)}\right\|_{w_{1}^{2-r}(D)}\right\} .
$$

Since $\operatorname{dist}(D, y) \geqslant 1 / 2 d$, it follows from (4.9) that

$$
\left|G^{(y)}\right|_{\infty}^{r(D)} \leqslant C d^{-N-r+2} .
$$

We shall next estimate the second term on the right in (6.5). We have

$$
\left\|e^{(y)}\right\|_{w_{1}^{2-r}(D)}=\sup _{\varphi \in C^{\infty}(D)} \frac{\left(e^{(y)}, \varphi\right)}{\|\varphi\|_{w_{\infty}^{r-2}(D)}} .
$$

Let $v \in W_{\infty}^{1}(D)$ satisfy $A(w, v)=(w, \varphi)$ for all $w \in W_{1}^{1}(D)$. Then

$$
\left(e^{(y)}, \varphi\right)=A\left(e^{(y)}, v\right)=v(y)-v_{h}(y),
$$

where $v_{n} \in S^{n}(D)$ satisfies $A\left(\chi, v-v_{n}\right)=0$ for all $\chi \in S^{n}(D)$. Since $y \in \Omega_{1}$, we may apply Corollary 5.1 to $\left(v-v_{h}\right)(y)$ on the spheres $D_{1}^{\prime} \subset \subset D^{\prime}$ of diameter $d / 8$ and $d / 4$, respectively, with center at $y$. Then

$$
\left|\left(v-v_{h}\right)(y)\right| \leqslant C\left\{h^{r}\left(\ln \left(\frac{d}{h}\right)\right)^{\bar{r}}|v|_{w_{\infty}^{r}\left(D^{\prime}\right)}+d^{-N / 2}\left\|_{v}-v_{h}\right\|_{0,0}\right\} .
$$

Note that $\operatorname{dist}\left(D^{\prime}, D\right) \geqslant d / 4$. Using the fact that $\varphi \in \mathcal{C}^{\infty}(D)$, we have, cf. the proof of Lemma 4.2 , that

$$
\|v\|_{w_{\infty}^{r}\left(D^{\prime}\right)} \leqslant C\left\|_{\varphi}\right\|_{w_{\infty}^{r-2}(D)} .
$$

Furthermore, from (6.2), cf. Lemma 4.3,

$$
\left\|v-v_{h}\right\|_{0, D} \leqslant C h^{r}\left\|_{\varphi-2, D} \leqslant C h^{r} d^{N / 2}\right\| \varphi \|_{w_{\infty}^{r-2}(D)}
$$

Hence

$$
\left|\left(e^{(y)}, \varphi\right)\right| \leqslant C h^{r}\left(\ln \left(\frac{d}{h}\right)\right)^{\bar{r}}\|\varphi\|_{w_{\infty}^{r-2}(D)} .
$$

This together with (6.7), (6.6) and (6.5) proves (6.3).

We shall next show (6.4). Let $d=|x-y| \leqslant C_{7} h$. In view of (4.9) it suffices to show that

$$
\left|G_{h}^{(y)}(x)\right| \leqslant \begin{cases}C \ln \frac{1}{h} & \text { for } N=2 \\ C h^{2-N} & \text { for } N \geqslant 3\end{cases}
$$

We define $x_{0}$ by 


$$
\sup _{x \in \Omega_{2}}\left|G_{h}^{(y)}(x)\right|=\left|G_{h}^{(y)}\left(x_{0}\right)\right| .
$$

We may assume that $B_{k n}\left(x_{0}\right) \subseteq \Omega_{2}$; otherwise $\left|x_{0}-y\right| \geqslant 1 / 2 \operatorname{dist}\left(\Omega_{1}, \Omega_{2}\right)$ and (6.8) follows from (6.3) and (4.9).

Let $A_{D}$ denote the form $A$ with the integration extended over the domain $D$. We have with $\epsilon$ to be chosen later,

$$
\begin{aligned}
G_{h}^{(y)}\left(x_{0}\right) & =A\left(G_{h}^{\left(x_{0}\right)}, G_{h}^{(y)}\right)=A\left(G^{\left(x_{0}\right)}, G_{h}^{(y)}\right) \\
& =A_{B_{\epsilon h}\left(x_{0}\right)}\left(G^{\left(x_{0}\right)}, G_{h}^{(y)}\right)+A_{D \backslash B_{\epsilon h}\left(x_{0}\right)}\left(G^{\left(x_{0}\right)}, G_{h}^{(y)}\right)=I_{1}+I_{2}
\end{aligned}
$$

Using the inverse property (2.2.b) and (4.9), we have

$$
\begin{aligned}
& I_{1} \leqslant C\left\|G_{h}^{(y)}\right\|_{W_{\infty}^{1}\left(B_{\epsilon h}\left(x_{0}\right)\right)}\left\|G^{\left(x_{0}\right)}\right\|_{W_{1}^{1}\left(B_{\epsilon h}\left(x_{0}\right)\right)} \\
& \leqslant \frac{C}{h}\left|G_{h}^{(y)}\left(x_{0}\right)\right| \cdot \epsilon h=C \epsilon\left|G_{h}^{(y)}\left(x_{0}\right)\right| .
\end{aligned}
$$

For $I_{2}$, the Cauchy-Schwarz inequality and (4.9) give

$$
\begin{aligned}
I_{2} & \leqslant C \| G^{\left(x_{0}\right)\left\|_{1, D \backslash B_{\epsilon h}\left(x_{0}\right)}\right\| G_{h}^{(y)} \|_{1, D}} \\
& \leqslant \begin{cases}C(\epsilon)(\ln 1 / h)^{1 / 2}\left\|G_{h}^{(y)}\right\|_{1,0} & \text { for } N=2, \\
C(\epsilon) h^{-N / 2+1}\left\|G_{h}^{(y)}\right\|_{1, D} & \text { for } N \geqslant 3 .\end{cases}
\end{aligned}
$$

Note that by (6.1) and (6.9),

$$
\left\|G_{h}^{(y)}\right\|_{1,0}^{2} \leqslant C A\left(G_{h}^{(y)}, G_{h}^{(y)}\right)=C G_{h}^{(y)}(y) \leqslant C\left|G_{h}^{(y)}\left(x_{0}\right)\right| ;
$$

thus

$$
I_{2} \leqslant \begin{cases}\widetilde{C}(\epsilon) \ln \frac{1}{h}+\epsilon\left|G_{h}^{(y)}\left(x_{0}\right)\right| & \text { for } N=2, \\ \widetilde{C}(\epsilon) h^{-N+2}+\epsilon\left|G_{h}^{(y)}\left(x_{0}\right)\right| & \text { for } N \geqslant 3 .\end{cases}
$$

Choosing $\epsilon$ small enough we obtain the desired inequality (6.8) from (6.9)-(6.12).

This completes the proof of the theorem.

Appendix 1. Proof of Theorem 5.1 for Noncoercive Forms $A$. Assume that we have proven the following weaker version of Theorem 5.1.

Lemma A.1. Under the assumptions of Theorem 5.1, if $u \in W_{\infty}^{1}(D)$ and

$$
A\left(u-u_{h}, \chi\right)=0 \text { for } \chi \in \AA^{n}(D),
$$

then

$$
\left\|u-u_{h}\right\|_{L_{\infty}(1 / 2 D)} \leqslant C\left(\|u\|_{w_{\infty}^{1}(D)}+\left\|u-u_{h}\right\|_{w_{q}^{-p}(D)}\right) .
$$

We shall first show how Theorem 5.1 follows from this. Let $K$ be a positive number such that the form $A_{K}, A_{K}(v, w) \equiv A(v, w)+K(v, w)$ is coercive, i.e., satisfies (4.1). Let $\omega \in \mathcal{C}^{\infty}(1 / 2 D), \omega \equiv 1$ on $1 / 4 D$, let $\tilde{u}=\omega u$ and let $\tilde{u}_{h} \in S^{h}(D)$ be 
given by

$$
A_{K}\left(\tilde{u}-\tilde{u}_{n}, \chi\right)=0 \text { for } \chi \in S^{n}(D)
$$

By Lemma 5.1,

$$
\left\|\tilde{u}-\tilde{u}_{h}\right\|_{L_{\infty}(1 / 4 D)} \leqslant C\left(\ln \frac{1}{h}\right)^{\bar{r}}\|u\|_{L_{\infty}(D)}
$$

Next note that

$$
A\left(\tilde{u}_{n}-u_{n}, \chi\right)=-K\left(\tilde{u}_{n}-\tilde{u}, \chi\right) \text { for } \chi \in \xi^{n}(1 / 4 D)
$$

For $D^{\prime}=c D$ with sufficiently small $c$, the form $A$ is coercive over $\stackrel{\circ}{H}^{1}\left(D^{\prime}\right)$. Let then $\psi \in \stackrel{\circ}{H}^{1}\left(D^{\prime}\right)$ be such that

$$
A(\psi, v)=K\left(\tilde{u}_{n}-\tilde{u}, v\right) \quad \text { for } v \in \stackrel{\circ}{H}^{1}\left(D^{\prime}\right) .
$$

Thus,

$$
A\left(\psi-\left(u_{h}-\tilde{u}_{h}\right), \chi\right)=0 \text { for all } \chi \in S^{n}\left(D^{\prime}\right)
$$

and hence using Lemma A.1 and (A.2),

$$
\begin{aligned}
& \left\|\psi-\left(u_{h}-\tilde{u}_{h}\right)\right\|_{L_{\infty}\left(1 / 4 D^{\prime}\right)} \\
& \quad \leqslant C\|\psi\|_{w_{\infty}^{1}\left(D^{\prime}\right)}+C\left\|\psi-\left(u_{h}-\tilde{u}_{h}\right)\right\|_{w_{q}^{-p}\left(D^{\prime}\right)} \\
& \quad \leqslant C\|\psi\|_{w_{\infty}^{1}\left(D^{\prime}\right)}+C\left\|_{h}-u\right\|_{w_{q}^{-p}(1 / 4 D)}+C(\ln 1 / h)^{\bar{r}} \|_{u \|_{L_{\infty}(D)}} .
\end{aligned}
$$

Thus, by (A.2) and the triangle inequality,

$$
\left\|u-u_{h}\right\|_{L_{\infty}\left(1 / 4 D^{\prime}\right)} \leqslant C\|\psi\|_{w_{\infty}^{1}\left(D^{\prime}\right)}+C\left\|_{h}-u\right\|_{w_{q}^{-p}(1 / 4 D)}+C\left(\ln \frac{1}{h}\right)^{\bar{r}} \|_{u \|_{L_{\infty}(D)}}
$$

By elliptic regularity, cf. Lemma 4.1, and (A.2),

$$
\|\psi\|_{w_{\infty}^{1}\left(D^{\prime}\right)} \leqslant C\left\|\tilde{u}_{h}-\tilde{u}\right\|_{L_{\infty}\left(D^{\prime}\right)} \leqslant C\left(\ln \frac{1}{h}\right)^{\bar{r}}\|u\|_{L_{\infty}(D)},
$$

so that finally

$$
\left\|u-u_{h}\right\|_{L_{\infty}\left(1 / 4 D^{\prime}\right)} \leqslant C\left(\ln \frac{1}{h}\right)^{\bar{r}}\left\|_{u}\right\|_{L_{\infty}(D)}+C\left\|u-u_{h}\right\|_{w_{q}^{-p}(D)}
$$

Theorem 5.1 follows from this.

It remains to prove Lemma 4.1. We shall, under the general assumptions of Theorem 5.1, prove the following:

Lemмa A.2. Let $D^{\prime} \subset \subset D$ be concentric spheres. If $2 \leqslant q<p \leqslant \infty$ with $1 / q$ $-1 / p<1 / N$, then

$$
\left\|u-u_{h}\right\|_{L_{p}\left(D^{\prime}\right)} \leqslant C\left(\|u\|_{w_{\infty}^{1}(D)}+\left\|u-u_{h}\right\|_{L_{q}(D)}\right) .
$$

Lemma A.1 follows from Lemma A.2, for, by iteration of Lemma A.2 one has

$$
\left\|u-u_{h}\right\|_{L_{\infty}(1 / 4 D)} \leqslant C\left\|_{w_{\infty}(1 / 2 D)}+C\right\|_{u}-u_{h} \|_{L_{2}(1 / 2 D)} \text {. }
$$


By Lemma 3.1,

$$
\left\|u-u_{h}\right\|_{L_{2}(1 / 2 D)} \leqslant C\left\|_{w_{\infty}(D)}^{1}+\right\| u-u_{h} \|_{w_{p}^{-q}(D)},
$$

and thus, Lemma A.1 obtains.

Proof of Lemma A.2. Let $K$ be such that $A_{K}$ is coercive. Now,

$$
A_{K}\left(u-u_{n}, \chi\right)=K\left(u-u_{n}, \chi\right) \text { for } \chi \in S^{n}(D) .
$$

Let $\psi$ be such that

$$
A_{K}(\psi, v)=K\left(u-u_{h}, v\right) \quad \text { all } v \in H^{1}(D),
$$

and $\psi_{n} \in S^{h}(D)$ such that $A_{K}\left(\psi-\psi_{h}, \chi\right)=0$ for all $\chi \in S^{h}(D)$. Note that

$$
A_{K}\left(u-u_{n}-\psi_{h}, \chi\right)=0 \text { for } \chi \in \AA^{n}(D),
$$

and we may apply Theorem 5.1 in the coercive case to deduce

$$
\left\|u-u_{h}-\psi_{h}\right\|_{L_{\infty}\left(D^{\prime}\right)} \leqslant C\left\|_{u}\right\|_{w_{\infty}^{1}(D)}+C\left\|_{u}-u_{h}\right\|_{0, D}+C\left\|\psi_{h}\right\|_{0, D} .
$$

Since

$$
\left\|\psi_{n}\right\|_{0, D} \leqslant C\left\|\psi_{n}\right\|_{1, D} \leqslant C\left\|_{u}-u_{h}\right\|_{0, D}
$$

and

$$
\left\|u-u_{h}\right\|_{L_{p}\left(D^{\prime}\right)} \leqslant\left\|u-u_{h}-\psi_{h}\right\|_{L_{p}\left(D^{\prime}\right)}+\left\|\psi_{h}\right\|_{L_{p}\left(D^{\prime}\right)},
$$

the desired result will follow if we can show that

(A.3) $\left\|\psi_{n}\right\|_{L_{p}\left(D^{\prime}\right)} \leqslant C\left\|_{u}-u_{n}\right\|_{L_{q}(D)}$ for $2 \leqslant p \leqslant \infty, 0<1 / q-1 / p<1 / N$.

Since

$$
\left\|\psi-\psi_{n}\right\|_{0, D} \leqslant\left\|\psi-\psi_{n}\right\|_{1, D} \leqslant C\|\psi\|_{1, D} \leqslant C\|\psi\|_{w_{\infty}^{1}(D)},
$$

we obtain using Sobolev's lemma and elliptic regularity,

$$
\begin{aligned}
& \left\|\psi_{h}\right\|_{L_{\infty}\left(D^{\prime}\right)} \leqslant\left\|\psi-\psi_{h}\right\|_{L_{\infty}\left(D^{\prime}\right)}+\|\psi\|_{L_{\infty}\left(D^{\prime}\right)} \\
& \leqslant C h\left(\ln \frac{1}{h}\right)^{\bar{r}}\|\psi\|_{W_{\infty}^{1}(D)}+C\left\|\psi-\psi_{h}\right\|_{0, D}+C\|\psi\|_{L_{\infty}\left(D^{\prime}\right)} \\
& \leqslant C\|\psi\|_{w_{\infty}^{1}(D)} \leqslant C\|\psi\|_{w_{s}^{2}(D)} \leqslant C\left\|_{u}-u_{h}\right\|_{L_{s}(D)} \quad \text { for } 1 / s<1 / N .
\end{aligned}
$$

Similarly,

$$
\begin{aligned}
\left\|\psi_{h}\right\|_{0, D^{\prime}} & \leqslant\|\psi\|_{1, D} \leqslant C\|\psi\|_{w_{s}^{2}(D)} \\
& \leqslant C\left\|u-u_{h}\right\|_{L_{s}(D)} \text { for } 1 / s-1 / 2<1 / N .
\end{aligned}
$$

By interpolation between (A.4) and (A.5) we obtain (A.3). As noted, this proves Lemma A.2.

This completes the proof of Theorem 5.1 in the case of noncoercive forms. 
Appendix 2. Proof of (0.7). As mentioned in the Introduction, we shall take the form $A$ to be coercive, i.e., satisfy (4.1). We assume that A.1, A.2 and A.3 hold (see Section 2), and furthermore that $S^{h}(\Omega) \subseteq H^{2}(\Omega)$. We shall then prove that if $A\left(u-u_{n}, \chi\right)=0$ for all $\chi \in S^{h}(\Omega)$, then for $\Omega_{1} \subset \subset \Omega, p \geqslant 0,1 \leqslant q \leqslant \infty$,

(0.7) $\left\|u-u_{h}\right\|_{L_{2}\left(\Omega_{1}\right)} \leqslant C\left(\|u-\chi\|_{L_{2}(\Omega)}+\left\|u-u_{h}\right\|_{w_{q}^{-p}(\Omega)}\right)$ for any $\chi \in S^{h}(\Omega)$.

Let $\Omega_{1} \subset \subset \Omega_{2} \subset \subset \Omega_{3} \subset \subset \Omega_{4} \subset \subset \Omega$ where we may assume that $\partial \Omega_{4}$ is smooth. Let $\omega \in \mathcal{C}^{\infty}\left(\Omega_{3}\right)$ with $\omega \equiv 1$ on $\Omega_{2}$, and put $\tilde{u}=\omega u$. Let $\tilde{u}_{n} \in S^{h}\left(\Omega_{4}\right)$ satisfy $A\left(\tilde{u}-\tilde{u}_{h}, \chi\right)=0$ for all $\chi \in S^{h}\left(\Omega_{4}\right)$, where the form $A$ is now taken over $\Omega_{4}$. Then

$$
\left\|u-u_{h}\right\|_{0, \Omega_{1}} \leqslant\left\|\tilde{u}-\tilde{u}_{h}\right\|_{0, \Omega_{1}}+\left\|\tilde{u}_{h}-u_{h}\right\|_{0, \Omega_{1}} .
$$

We shall estimate the two terms on the right-hand side. We have

$$
\left\|\tilde{u}-\tilde{u}_{n}\right\|_{0, \Omega_{4}}=\sup _{\varphi \in \check{C}^{\infty}\left(\Omega_{4}\right)} \frac{\left|\left(\tilde{u}-\tilde{u}_{n}, \varphi\right)\right|}{\|\varphi\|_{0, \Omega_{4}}} .
$$

For each such $\varphi$ let $v \in H^{2}\left(\Omega_{4}\right)$ satisfy $A(\psi, v)=(\psi, \varphi)$ for all $\psi \in H^{1}\left(\Omega_{4}\right)$. Hence if $v_{h} \in S^{h}\left(\Omega_{4}\right)$ satisfies $A\left(\chi, v-v_{h}\right)=0$ for all $\chi \in S^{h}\left(\Omega_{4}\right)$, we have

$$
\left(\tilde{u}-\tilde{u}_{h}, \varphi\right)=A\left(\tilde{u}-\tilde{u}_{h}, v\right)=A\left(\tilde{u}, v-v_{h}\right) .
$$

Integrating by parts and using Schwarz' inequality, we obtain

$$
\left|\left(\tilde{u}-\tilde{u}_{h}, \varphi\right)\right|=\left|\left(\tilde{u}, L^{*}\left(v-v_{h}\right)\right)\right| \leqslant C\|\tilde{u}\|_{0, \Omega_{3}}\left\|v-v_{h}\right\|_{2, \Omega_{3}} .
$$

For a suitable $\chi \in S^{h}\left(\Omega_{4}\right)$, given by Proposition 2.1, we have on using the inverse property A.2 and the fact that $\left\|v-v_{h}\right\|_{1, \Omega_{4}} \leqslant C \inf _{\chi \in S^{n}\left(\Omega_{4}\right)}\|v-\chi\|_{1, \Omega_{4}}$,

$$
\begin{aligned}
\| v & v_{h}\left\|_{2, \Omega_{3}} \leqslant\right\|_{v}-\chi\left\|_{2, \Omega_{3}}+\right\| \chi-v_{h} \|_{2, \Omega_{3}} \\
& \leqslant\left\|_{v}-\chi\right\|_{2, \Omega_{3}}+C h^{-1}\left\|_{\chi}-v_{h}\right\|_{1, \Omega_{4}} \\
& \leqslant\left\|_{v}-\chi\right\|_{2, \Omega_{3}}+C h^{-1}\left\|_{v}-\chi\right\|_{1, \Omega_{4}}+C h^{-1}\left\|_{v}-v_{h}\right\|_{1, \Omega_{4}} \\
& \leqslant\left\|_{v}-\chi\right\|_{2, \Omega_{3}}+C h^{-1}\left\|_{v}-\chi\right\|_{1, \Omega_{4}} \\
& \leqslant C\left\|_{v}\right\|_{2, \Omega_{4}} \leqslant C\left\|_{\varphi}\right\|_{0, \Omega_{4}} .
\end{aligned}
$$

In the last step we used elliptic regularity, cf. Lemma 4.1. It follows from (A.7), (A.8) and (A.9) that

$$
\left\|\tilde{u}-\tilde{u}_{h}\right\|_{0, \Omega_{4}} \leqslant C\|u\|_{0, \Omega_{4}} \leqslant C\left\|_{u}\right\|_{0, \Omega} .
$$

For the second term on the right of (A.6), we note that $A\left(u_{n}-\tilde{u}_{n}, \chi\right)=0$ for all $\chi \in S^{n}\left(\Omega_{2}\right)$. By (3.4) and (A.10) (and assuming without loss of generality that $q \leqslant 2$ below), we have 


$$
\begin{aligned}
\left\|\tilde{u}_{h}-u_{h}\right\|_{0, \Omega_{1}} & \leqslant C\left\|\tilde{u}_{n}-u_{n}\right\|_{w_{q}^{-p}\left(\Omega_{2}\right)} \\
& \leqslant C\left(\left\|\tilde{u}-\tilde{u}_{h}\right\|_{0, \Omega_{2}}+\left\|u-u_{n}\right\|_{w_{q}^{-p}(\Omega)}\right) \\
& \leqslant C\left(\|u\|_{0, \Omega}+\left\|u-u_{n}\right\|_{w_{q}^{-p}(\Omega)}\right) .
\end{aligned}
$$

Inserting (A.10) and (A.11) into (A.6) we obtain the result (0.7) with $\chi=0$. The general case follows by writing $u-u_{n}=(u-\chi)-\left(u_{n}-\chi\right)$. This completes the proof of (0.7).

Department of Mathematics

Cornell University

Ithaca, New York 14853

1. I. BABUŠKA \& A. K. AZIZ, "Survey lectures on the mathematical foundations of the finite element method," The Mathematical Foundations of the Finite Element Method with Applications to Partial Differential Equations, Part I (A. K. Aziz, Editor), Academic Press, New York, 1972, pp. 1-359. MR 49 \#11824.

2. J. H. BRAMBLE, "A survey of some finite element methods proposed for treating the Dirichlet problem," Advances in Math., v. 16, 1975, pp. 187-196. MR 52 \#245.

3. J. H. BRAMBLE, J. A. NITSCHE \& A. H. SCHATZ, "Maximum-norm interior estimates for Ritz-Galerkin methods," Math. Comp., v. 29, 1975, pp. 677-688.

4. J. H. BRAMBLE \& J. E. OSBORN, "Rate of convergence estimates for nonselfadjoint eigenvalue approximations," Math. Comp., v. 27, 1973, pp. 525-549. MR 51 \#280.

5. J. H. BRAMBLE \& A. H. SCHATZ, "Estimates for spline projections," Rev. Française Automat. Informat. Recherche Opérationelle Sér. Rouge, v. 10, 1976, pp. 5-37.

6. J. H. BRAMBLE \& V. THOMÉE, "Interior maximum norm estimates for some simple finite element methods," Rev. Française Automat. Informat. Recherche Opérationelle Sér. Rouge, v. 8, 1974, pp. 5-18. MR 50 \#11808.

7. J. H. BRAMBLE \& M. ZLÁMAL, "Triangular elements in the finite element method," Math. Comp., v. 24, 1970, pp. 809-820. MR 43 \#8250.

8. P. G. CIARLET \& P. A. RAVIART, "General Lagrange and Hermite interpolation in $R^{n}$ with applications to finite element methods," Arch. Rational Mech. Anal., v. 46, 1972, pp. 177-199. MR 49 \#1730.

9. P. G. CIARLET \& P. A. RAVIART, "Maximum principle and uniform convergence for the finite element method," Comput. Methods Appl. Mech. Engrg., v. 2, 1973, pp. 17-31. MR 51 \#1 1992.

10. J. DOUGLAS, JR., T. DUPONT \& L. WAHLBIN, “Optimal $L_{\infty}$ error estimates for Galerkin approximations to solutions of two-point boundary value problems," Math. Comp., v. 29, 1975, pp. 475-483. MR 51 \#298.

11. S. HILBERT, "A mollifier useful for approximations in Sobolev spaces and some applications to approximating solutions of differential equations," Math. Comp., v. 27, 1973, pp. 81-89. MR 48 \#10047.

12. F. JOHN, "General properties of solutions of linear elliptic partial differential equations," Proc. Sympos. on Spectral Theory and Differential Problems, Oklahoma A \& M College, Stillwater, Okla., 1951, pp. 113-175. MR 13, 349.

13. C. MIRANDA, Partial Differential Equations of Elliptic Type, 2nd rev. ed., SpringerVerlag, Berlin and New York, 1970.

14. F. NATTERER, "Über die punktweise Konvergenz Finiter Elemente," Numer. Math., v. 25,1975, pp. $67-77$.

15. J. A. NITSCHE, “ $L_{\infty}$-convergence for finite element approximation," 2 nd Conf. on

Finite Elements (Rennes, France, May 12-14, 1975).

16. J. A. NITSCHE \& A. H. SCHATZ, "Interior estimates for Ritz-Galerkin methods," Math. Comp., v. 28, 1974, pp. 937-958. MR 51 \#525. 
17. M. SCHECHTER, "On $L^{p}$ estimates and regularity. I," Amer. J. Math., v. 85, 1963, pp. 1-13. MR 32 \#6051.

18. R. SCOTT, "Optimal $L^{\infty}$ estimates for the finite element method on irregular meshes," Math. Comp., v. 30, 1976, pp. 681-697.

19. G. STRANG, "Approximation in the finite element method," Numer. Math., v. 19, 1972, pp. 81-98. MR 46 \#4677.

20. G. STRANG \& G. FIX, "A Fourier analysis of the finite element variational method." (Unpublished manuscript.)

21. M. F. WHEELER, "An optimal $L_{\infty}$ error estimate for Galerkin approximations to solutions of two-point boundary value problems," SIAM J. Numer. Anal., v. 10, 1973, pp. 914-917. MR 49 \#8399. 